\title{
Dlg1, Sec8, and Mtmr2 Regulate Membrane Homeostasis in Schwann Cell Myelination
}

\author{
Annalisa Bolis, ${ }^{1,2}$ Silvia Coviello, ${ }^{1,2}$ Ilaria Visigalli, ${ }^{3}$ Carla Taveggia, ${ }^{2}$ Angela Bachi, ${ }^{4}$ Athar H. Chishti, ${ }^{5}$ \\ Toshihiko Hanada, ${ }^{5}$ Angelo Quattrini, ${ }^{2}$ Stefano Carlo Previtali, ${ }^{2}$ Alessandra Biffi, ${ }^{3}$ and Alessandra Bolino ${ }^{1,2}$ \\ ${ }^{1}$ Dulbecco Telethon Institute, ${ }^{2}$ INSPE-Institute of Experimental Neurology, ${ }^{3}$ San Raffaele Telethon Institute for Gene Therapy, and ${ }^{4}$ Biological Mass \\ Spectrometry, San Raffaele Scientific Institute, 20132 Milan, Italy, and ${ }^{5}$ Department of Pharmacology, University of Illinois College of Medicine, Chicago, \\ Illinois 60612
}

How membrane biosynthesis and homeostasis is achieved in myelinating glia is mostly unknown. We previously reported that loss of myotubularin-related protein 2 (MTMR2) provokes autosomal recessive demyelinating Charcot-Marie-Tooth type 4B1 neuropathy, characterized by excessive redundant myelin, also known as myelin outfoldings. We generated a Mtmr2-null mouse that models the human neuropathy. We also found that, in Schwann cells, Mtmr2 interacts with Discs large 1 (Dlg1), a scaffold involved in polarized trafficking and membrane addition, whose localization in Mtmr2-null nerves is altered. We here report that, in Schwann cells, Dlg1 also interacts with kinesin 13B (kif13B) and Sec8, which are involved in vesicle transport and membrane tethering in polarized cells, respectively. Taking advantage of the Mtmr2-null mouse as a model of impaired membrane formation, we provide here the first evidence for a machinery that titrates membrane formation during myelination. We established Schwann cell/DRG neuron cocultures from Mtmr2-null mice, in which myelin outfoldings were reproduced and almost completely rescued by Mtmr2 replacement. By exploiting this in vitro model, we propose a mechanism whereby kif13B kinesin transports Dlg1 to sites of membrane remodeling where it coordinates a homeostatic control of myelination. The interaction of Dlg1 with the Sec8 exocyst component promotes membrane addition, whereas with Mtmr2, negatively regulates membrane formation. Myelin outfoldings thus arise as a consequence of the loss of negative control on the amount of membrane, which is produced during myelination.

\section{Introduction}

Myelin is a multilamellar membrane, which provides electrical insulation around the axon and participates to bidirectional communication with neurons and the extracellular environment. This structure is produced by oligodendrocytes in the CNS and by Schwann cells in the PNS (Trapp and Kidd, 2004). In both cell types, myelin biogenesis involves sorting and recruitment of selected lipids, proteins, and mRNAs to specific subdomains, with a strong analogy to polarized transport in epithelial cells and neuronal synapses (Sherman and Brophy, 2005; Trajkovic et al., 2006; Simons and Trotter, 2007). However, the molecular basis for sorting and polarized transport in glial cells is mostly unknown.

Charcot-Marie-Tooth type 4B1 (CMT4B1) is an autosomal

\footnotetext{
Received March 25, 2009; revised May 20, 2009; accepted June 1, 2009.

This work was supported by the Italian Telethon, Association Française contre les Myopathies, E-Rare (ERA-Net for research programs on rare diseases), Compagnia San Paolo, and Istituto Superiore di Sanità (A. Bolino). A. Bolino is a recipient of a Telethon Career Award. C.T. is supported by Fondazione Italiana Sclerosi Multipla cod 2007/PC/01. We thank Drs. P. Brophy and D. Sherman for providing the nerve CDNA library, Dr. S. Hsu for the generous gift of Sec 8 plasmid and antibody, Dr. T. Nakagawa for the anti-Dlg1 antibody, Dr. E. Peles for the anti-Caspr antibody, and Dr. V. Lee for the anti-MBP antibody. We are indebted to Dr. J. Salzer for providing the protocol for the coculture assessment. We also thank Dr.P. Podini for her help with electron microscopy. We are also indebted to Drs. De Camilli, M. L. Feltri, and L. Wrabetz for comments and critical reading of this manuscript. A. Bolino wishes to dedicate this paper to the memory of Mario Bolino.

Correspondence should be addressed to Dr. Alessandra Bolino, Dulbecco Telethon Institute and INSPE-Institute of Experimental Neurology, San Raffaele Scientific Institute, 20132 Milan, Italy. E-mail: bolino.alessandra@hsr.it. D0I:10.1523/JNEUROSCI.1423-09.2009

Copyright $\odot 2009$ Society for Neuroscience $\quad$ 0270-6474/09/298858-13\$15.00/0
}

recessive demyelinating neuropathy with childhood onset caused by loss of the myotubularin-related protein 2 (MTMR2) phospholipid phosphatase (Bolino et al., 2000; Bolis et al., 2007; Previtali et al., 2007). The hallmark of the pathology is the presence of redundant loops of myelin around large myelinated axons in nerve, also known as "myelin outfoldings." We previously generated a Mtmr2-null mouse that reproduces the neuropathy (Bolino et al., 2004), and we reported that Mtmr2 Schwann cellspecific ablation is sufficient and necessary to cause the dysmyelination (Bolis et al., 2005). In mutant nerves, redundant loops of myelin arise after the third postnatal week mainly at paranodal regions, known sites for membrane remodeling, suggesting that the loss of Mtmr2 does not affect myelin formation per se but rather its control. Interestingly, we found that, in Schwann cells, Mtmr2 specifically interacts with Discs large 1 (Dlg1), also known as SAP97 (synapse-associated protein 97) (Bolino et al., 2004). Dlg1 is a multidomain scaffolding protein, which is involved in polarized trafficking and in membrane addition in Drosophila (Lee et al., 2003; Gorczyca et al., 2007). We previously reported that Dlg1 is found in myelin-forming Schwann cells, and it is enriched at Schwann cell paranodes, where myelin outfoldings preferentially arise (Bolis et al., 2005). In Mtmr2-null nerve fibers, this localization is altered, suggesting that the Mtmr2/Dlg1 interaction is relevant to the pathogenesis (Bolino et al., 2004; Bolis et al., 2005).

We therefore investigated whether loss of Mtmr2/Dlg1mediated control of membrane formation during Schwann cell 
myelination leads to excessive myelin with redundant folds. Here, we report that, in Schwann cells, the Dlg1 scaffold also interacts with kinesin 13B (kif13B) and Sec8, both involved in vesicular trafficking in polarized cells. We here provide evidence that in Schwann cells the kif13B motor protein (Hanada et al., 2000) transports Dlg1 to sites of membrane remodeling. We propose that the interaction of Dlg1 with the Sec8 exocyst component (Hsu et al., 2004) promotes membrane formation, whereas with Mtmr2, negatively regulates membrane addition. To support this hypothesis, we established Schwann cell/dorsal root ganglion (DRG) neuron cocultures from Mtmr2-null mice and we reproduced in vitro myelin outfoldings, which are rescued by Mtmr2 replacement. Consistently, myelin outfoldings are also rescued by reducing Sec 8 expression in Schwann cells. We here propose a novel mechanism that titrates membrane formation during Schwann cell myelination.

\section{Materials and Methods}

Mice. Mtmr2-null mice have been reported previously (Bolino et al., 2004). Teased fiber preparation and transverse sections from Mtmr2-null and wild-type mice have been performed as described previously (Bolino et al., 2004; Bolis et al., 2005).

Experiments with animals followed protocols approved by the Institutional Animal Care and Use Committees of our institutes.

Antibodies. For Western blot analysis, the following antibodies were used: rat anti-myelin basic protein (MBP) (provided by Dr. V. Lee, University of Pennsylvania, Philadelphia, PA); rabbit anti-NF-L (Millipore Bioscience Research Reagents); rabbit anti-FLAG (Sigma-Aldrich); mouse anti-FLAG (Sigma-Aldrich); mouse anti- $\beta$-tubulin (Sigma-Aldrich); mouse anti-Sec8 (BD Biosciences Transduction Laboratories) and mouse anti-Sec8 (Assay Designs); mouse anti-Dlg1 (Assay Designs), rabbit anti-Dlg1 (Affinity Bioreagents), and rabbit polyclonal anti-Dlg1 (provided by Dr. T. Nakagawa, University of California, San Diego, La Jolla, CA); mouse anti-kif13B and rabbit anti-kif13B (Dr. A. Chishti, University of Illinois College of Medicine, Chicago, IL); mouse anti-S100 (Sigma-Aldrich) and rabbit anti-S100 (Sigma-Aldrich); mouse antiGFAP (Millipore Bioscience Research Reagents) and rabbit anti-GFAP (Sigma-Aldrich); rabbit anti-NF-M (Millipore Bioscience Research Reagents); rabbit anti-NF-H (Millipore Bioscience Research Reagents); mouse anti-Caspr and rabbit anti-Caspr-GOGI (provided by Dr. E. Peles, The Weizmann Institute of Science, Rehovat, Israel); mouse anti$\mathrm{NaCh}$ Pan (Sigma-Aldrich); rabbit anti-ezrin (Sigma-Aldrich); mouse anti-E-cadherin (BD Biosciences Transduction Laboratories); rabbit anti-calnexin (Sigma-Aldrich); rabbit anti-Giantin (Covance); rabbit anti-Myc (Cell Signaling) and mouse anti-Myc (Santa Cruz Biotechnology); mouse anti-MTMR2 (Abnova); and mouse anti-green fluorescent protein (GFP) (Roche).

Glutathione S-transferase-binding assays. Glutathione S-transferase (GST) fusion proteins were expressed in Escherichia coli BL21 cells and purified directly from bacterial extract on glutathione-Sepharose 4 Fast Flow beads. Rat sciatic nerves and rat isolated Schwann cells were homogenated, and protein lysates were prepared using a binding buffer with $1 \%$ NP-40, 50 mu Tris buffer, pH 7.4, 10\% glycerol, $100 \mathrm{~mm} \mathrm{NaCl}, 10 \mathrm{~mm}$ $\mathrm{NaF}, 1 \mathrm{~mm} \mathrm{Na}$-vanadate. Equal amounts of protein lysates were incubated for $4 \mathrm{~h}$ at $4^{\circ} \mathrm{C}$ with immobilized GST fusion proteins and GST as control. After three washes with a buffer containing $0.5 \%$ NP-40, the bead pellets were dissolved in SDS sample buffer and analyzed by SDSPAGE and immunoblotting. To show the relative amount of GST fusion proteins used, bead pellets were dissolved again in SDS sample buffer and analyzed by SDS-PAGE, and the gel was colored with Coomassie.

Cell cultures. Myelin-forming Schwann cell/DRG neuron cocultures were established from embryonic day 13.5 mouse embryos as previously described (Previtali et al., 2003a; Taveggia et al., 2005) with minor modifications. Myelination was induced by treatment for $15 \mathrm{~d}$ with ascorbic acid (final concentration, $50 \mu \mathrm{g} / \mathrm{ml}$ ) (Sigma-Aldrich), with the exception of Dlg1 short hairpin RNA (shRNA) lentiviral vector (LV) depletion, in which myelination was analyzed at $4-7 \mathrm{~d}$ of ascorbic acid treatment.
Dissociated Schwann cell/DRG neuron cocultures were established as described but DRGs were first incubated with trypsin (0.25\%) for $45 \mathrm{~min}$ at $37^{\circ} \mathrm{C}$. Cells were also mechanically dissociated and then plated at a concentration of one to two DRGs per glass coverslip. Isolated rat Schwann cells were prepared as reported previously (Taveggia et al., 2005 ) and cultured using DMEM with $10 \%$ of fetal calf serum, $2 \mathrm{ng} / \mathrm{ml}$ recombinant human neuregulin1-b1 (R\&D Systems), and $2 \mathrm{~mm}$ forskolin (Calbiochem).

Transfection, preparation of tissues and cell lysates, and immunoprecipitation. Transfection of COS7 cells was performed using the SuperFect transfection reagent following manufacturer's condition (QIAGEN). Transiently transfected COS-7 cells were harvested $48 \mathrm{~h}$ after transfection, washed twice with cold PBS, pH 7.4, and lysed in buffer containing $1 \%$ NP-40, 150 mм NaCl, 50 mм Tris-HCl, pH 8.0, 10 mм NaF, 1 mм $\mathrm{Na}$-vanadate, and protease inhibitor mixture (Roche). After centrifugation at $13,000 \mathrm{rpm}$, the lysate was incubated with $5 \mathrm{mg} / \mathrm{ml} \mathrm{rabbit} \mathrm{anti-}$ Dlg1 antibody (Affinity Bioreagents) or rabbit IgG for control (SigmaAldrich). After $3 \mathrm{~h}$ incubation with the antibody at $4^{\circ} \mathrm{C}, 30 \mu \mathrm{l}$ of protein A-agarose (GE Healthcare) was added to the samples and incubated for at least $1 \mathrm{~h}$ at $4^{\circ} \mathrm{C}$. The agarose was washed three times with cold PBSTween $(0.1 \%)$, and the immunoprecipitated complex was resolved by SDS-PAGE.

For coimmunoprecipitation from nerve lysate, a similar protocol was used with minor modifications: postnatal day 11 (P11) mouse nerves were lysed in buffer containing 1\% Triton X-100 (TX-100), $150 \mathrm{~mm}$ $\mathrm{NaCl}, 50 \mathrm{~mm}$ Tris- $\mathrm{HCl}, \mathrm{pH}$ 8.0, $10 \mathrm{~mm} \mathrm{NaF}, 1 \mathrm{~mm}$ Na-vanadate, and protease inhibitor mixture (Roche); immunoprecipitation was performed with $5 \mathrm{mg}$ of mouse anti-Dlg1 antibody (Assay Designs) or mouse IgG for control.

Protein lysates from DRG explants, isolated rat Schwann cells, and mouse/rat sciatic nerves were prepared using a lysis buffer containing $1 \%$ TX-100, $50 \mathrm{~mm}$ Tris buffer, pH 8.0, $150 \mathrm{~mm} \mathrm{NaCl}, 10 \mathrm{~mm} \mathrm{NaF}, 1 \mathrm{~mm}$ $\mathrm{Na}$-vanadate, and Complete (Roche) protease inhibitors.

Electron microscopy. Electron microscopy on explants was performed as described previously (Bolino et al., 2004).

Lentiviral preparation and transduction. VSV (vesicular stomatitis virus)-pseudotyped LV stocks were produced by transient cotransfection of the transfer constructs pRRLsin.cPPT.hPGK.transgene1.Wpre (transgenes being the cDNA of FLAG-MTMR2, FLAG-MTMR2 $\triangle P D Z B D$, myc-kif13B/DMD, myc-kif13B/MBS, and GFP), the third-generation packaging constructs pMD2.Lg/p.RRE and pRSV.Rev, and the pMD2.G envelope construct in $293 \mathrm{~T}$ cells, followed by ultracentrifugation of conditioned medium, as described previously (Follenzi and Naldini, 2002). Stocks were titered by end-point integration titer in HeLa cells and quantified for particle content by HIV-1 Gag p24 immunocapture assay.

After 4-5 d after dissection, Schwann cell/DRG neuron cocultures were transduced by overnight incubation with the LV of interest at multiplicity of infection (MOI) 100, 50, 25, or 12.5. On the next day, cells were washed twice with C-media and supplemented with C-media for $2 \mathrm{~d}$. Then, myelination was induced as described above.

To downregulate KIF13B expression, a shRNAmir was used (Open Biosystems; clone ID, V2LMM_66797) and cloned into the pGIPZ LV [cytomegalovirus (CMV) promoter], which carried a GFP reporter. To downregulate expression of Sec8, a shRNA was used (Open Biosystems; clone ID, TRC_111728) cloned into the pLKO.1 LV (human U6 promoter), without a GFP reporter. To deplete Dlg1 expression, a shRNA was used (Open Biosystems; clone ID, TRCN0000025407) cloned into the pLKO.1 LV. Nonconcentrated LVs were used for RNA interference. The transfer constructs were transfected into 293FT cells together with packaging plasmids $\Delta 8.9$ and pCMV-VSGV using Lipofectamine 2000 (Invitrogen). As control, a vector encoding a shRNA to a nonspecific sequence (luciferase) was used. Viral supernatants were collected $72 \mathrm{~h}$ after transfection, centrifuged at $3000 \mathrm{rpm}$ for $15 \mathrm{~min}$, and frozen at $-80^{\circ} \mathrm{C}$. Using nonconcentrated LV, transduction of Schwann cell/DRG neuron cocultures was performed $4-5 \mathrm{~d}$ after dissection by incubating the cells with LVs for $24-48 \mathrm{~h}$. Cells were then supplemented with $\mathrm{C}$-media, and myelination was induced after $2 \mathrm{~d}$.

Quantitative PCR analysis was performed on genomic DNA and vector copies per genome were quantified by TaqMan analysis as described 
previously (Biffi et al., 2006). The number of LV copies per cell (vector copy number) was calculated as follows: (nanograms of LV/nanograms of endogenous DNA) $\times$ (number of LV integrations in the standard curve).

Immunocytochemistry. Immunofluorescence on cryosections was performed as described previously (Bolino et al., 2004). For immunohistochemistry, sciatic nerves were removed and rapidly snap-frozen in liquid nitrogen, either unfixed or previously fixed in buffered $4 \%$ paraformaldehyde. For teased-fiber preparation, sciatic nerves were removed, fixed on ice in freshly prepared buffered $4 \%$ paraformaldehyde, as described previously (Bolino et al., 2004). To compare protein expression and localization between Mtmr2-null and control fibers, images were acquired with the same exposure time, pinhole, and resolution were kept constant.

Schwann cell/DRG neuron cocultures were fixed for $15 \mathrm{~min}$ in $4 \%$ paraformaldehyde, permeabilized for $5 \mathrm{~min}$ in ice-cold methanol at $-20^{\circ} \mathrm{C}$, blocked for 20 min with $10 \%$ normal goat serum (Dako), 1\% bovine serum albumin (BSA) (Sigma-Aldrich), and then incubated with primary antibody for $1 \mathrm{~h}$. After extensive washing, the coverslips where incubated with the secondary antibody for $30 \mathrm{~min}$, washed, and mounted. For double immunostaining with anti-Dlg1 and anti-MBP antibody, the coverslips were blocked with $10 \%$ BSA for 20 min on ice, and incubation with mouse anti-Dlg1 antibody (Assay Designs) was performed for $1 \mathrm{~h}$ on ice. After incubations with an anti-mouse secondary antibody, rat anti-MBP and anti-rat secondary antibodies were performed at room temperature.

For immunolabeling, secondary antibodies included fluorescein-conjugated (FITC), rhodamine (tetramethylrhodamine isothiocyanate), or Cy5-conjugated donkey anti-mouse or -rabbit or -rat IgG (Jackson ImmunoResearch) and Alexa Fluor 488 goat anti-rabbit or anti-mouse IgG (Invitrogen). Slides and coverslips were analyzed using MRC 1024 laserscanning confocal (Bio-Rad), UltraVIEW ERS spinning disk confocal (PerkinElmer), TCS SP2 laser-scanning confocal (Leica) or Olympus BX (Olympus Optical) fluorescent microscope, and Zeiss Axiovert S100 TV2 with Hamamatsu OrcaII-ER.

Analysis of myelination. Myelination in Schwann cell/DRG neuron cocultures was evaluated as follows: using a fluorescence microscope, at least five fields per cover were randomly acquired near the sensory neurons, where usually myelination is more efficient and abundant and MBP-positive myelinated fibers were counted per field. Results were expressed as a percentage with respect to control values (percentage of myelination).

Myelination in dissociated Schwann cell/DRG neuron cocultures was evaluated as follows: using a fluorescence microscope, at least 10 fields per cover were randomly acquired and MBP-positive segments as well as Schwann cell nuclei were counted per field. Results were expressed as a ratio between MBP-positive segments and the Schwann cell nuclei.

To quantify MBP-positive fibers displaying myelin outfoldings, at least 200 MBP-positive myelinated fibers per explant were evaluated, in at least three different explants. The percentage of MBP-positive fibers showing myelin outfoldings on the total number of MBP-positive fibers was indicated.

Imaging and statistical analysis. Micrographs were acquired using a digital camera (Leica F300), and figures were prepared using Adobe Photoshop, version 8.0 (Adobe Systems).

Statistical analysis was performed using the Student $t$ test; two tails, unequal variants, and $\alpha=0.005$ were used. Error bars in the graphs represent SEM.

\section{Results}

Mtmr2-null Schwann cell/DRG neuron cocultures reproduce myelin outfoldings

We first assessed whether myelin outfoldings would be reproduced in vitro. To this aim, we established Schwann cell/DRG neuron cocultures from Mtmr2-null mice and we induced myelination by ascorbic acid treatment for $15 \mathrm{~d}$. Immunofluorescence staining for MBP, which labels compact myelin in the myelinated fibers, revealed irregularly shaped MBP-positive fibers, suggesting the presence of myelin outfoldings (Fig. $1 A-D$ ). These structures were further confirmed by electron microscopy (Fig. $1 E-$ $H$ ). Almost 75\% of MBP-positive myelinated fibers in Mtmr2null cocultures displayed myelin outfoldings (Fig. $2 D$ ). We never observed this morphology in either wild-type or heterozygous Mtmr2-null myelin-forming cocultures. In contrast to what we 

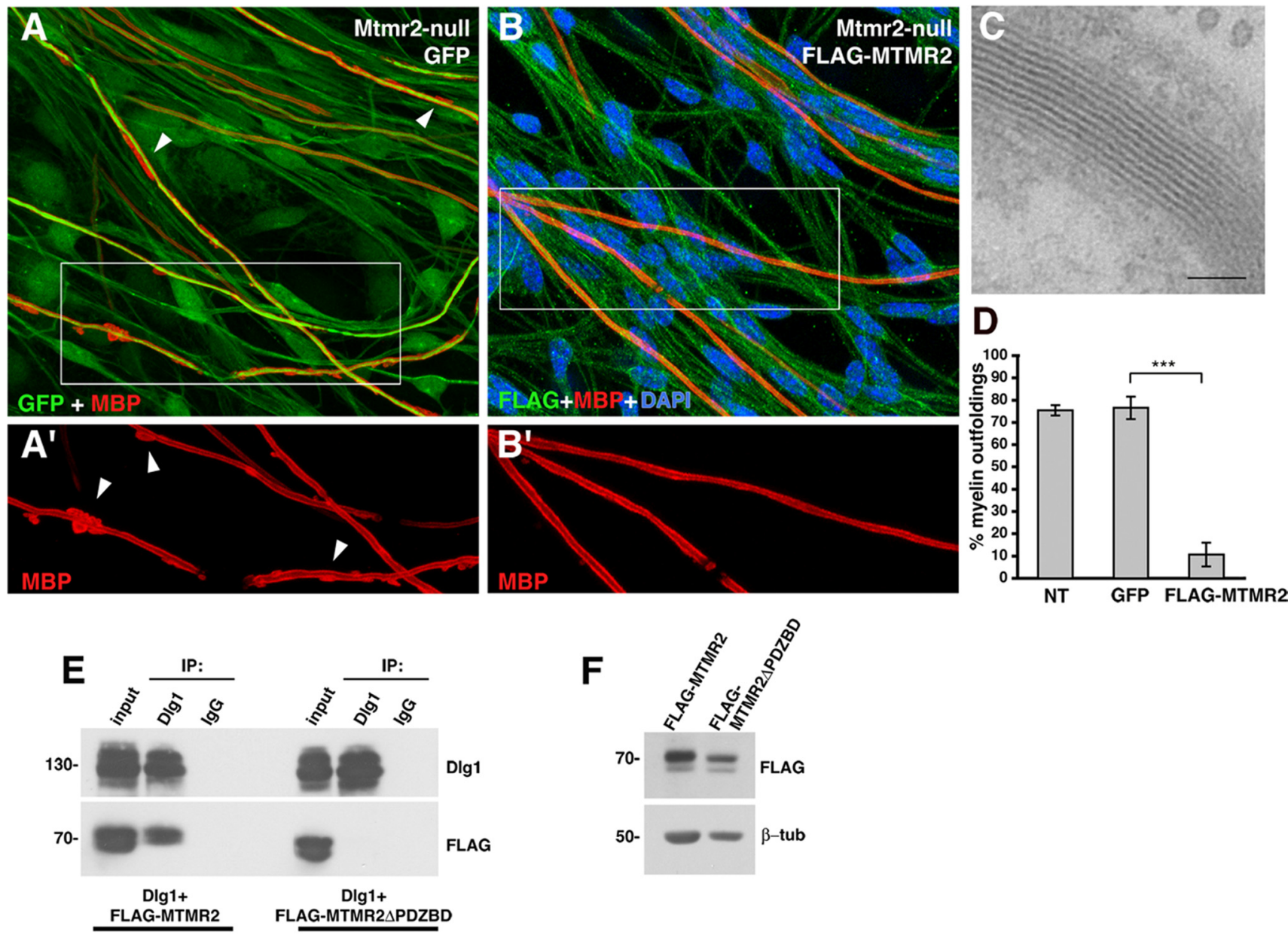

FLAG-MTMR2 FLAG-MTMR2 $\triangle$ PDZBD

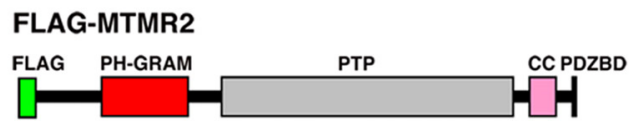

FLAG-MTMR2 $\triangle$ PDZBD
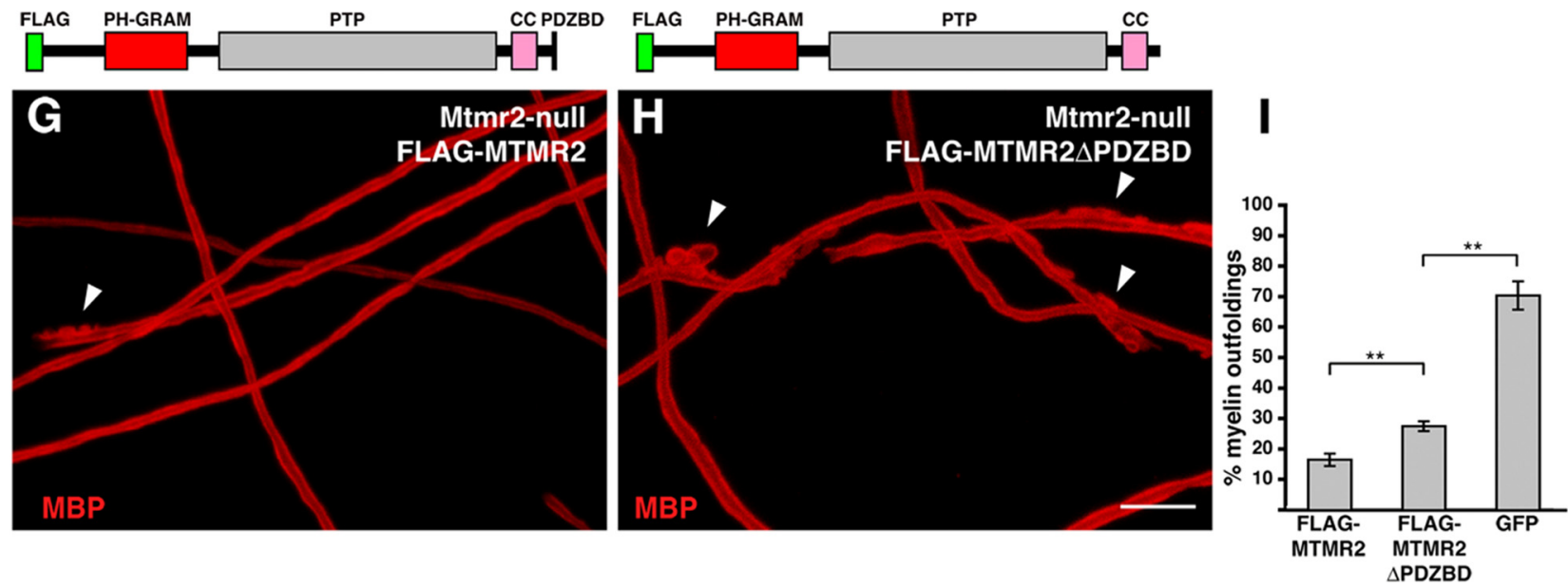

Figure 2. Loss of Mtmr2 and Mtmr2/Dlg1 interaction causes myelin outfoldings in vitro. Myelin outfoldings are rescued by MTMR2 replacement using LV transduction. $A$, Transduction of Mtmr2-null Schwann cell/DRG neuron cocultures with a GFP LV. $\boldsymbol{A}^{\prime}$, Inset, MBP-positive fibers with myelin outfoldings are indicated by arrowheads. $\boldsymbol{B}$, Transduction of $M$ tmr2-null Schwann cell/DRG neuron cocultures with FLAG-MTMR2 LV rescues myelin outfoldings. DAPI, 4', $6^{\prime}$-Diamidino-2-phenylindole. $\boldsymbol{B}^{\prime}$, Inset. C, Electron microscopy on FLAG-MTMR2 LV transduced cocultures showing that myelin compaction is preserved on MTMR2 overexpression. At least 30 myelinated fibers were evaluated and compared with cultures infected with GFP alone. $D$, Quantification of MBP-positive fibers with myelin outfoldings in not transduced (NT) explants $(75.5 \pm 2.3 ; n=6)$, transduced with GFP alone $(76.5 \pm 5.0 ; n=4)$, and with FLAG-MTMR2 $(10.7 \pm 5.3 ; n=9)\left(p=8.7 \times 10^{-6}\right)$ (from at least 2 different experiments). $E$, Dlg1 coimmunoprecipitates with FLAG-MTMR2 but not with MTMR2 devoid of the PDZ-binding domain at the C terminus, termed FLAG-MTMR2 $\triangle P D Z B D$. Input is the lysate. $\boldsymbol{F}$, Western blot analysis showing similar level of expression of both FLAG-MTMR2 and FLAG-MTMR2 $\triangle$ PDZBD on LV transduction of Mtmr2-null DRG explants at M0112.5. G, $\boldsymbol{H}$, Examples of myelin-forming cocultures transduced with FLAG-MTMR2 or FLAG-MTMR2 $\triangle P D Z B D ~ L V$ at MOI12.5. I, Quantification of MBP-positive fibers with myelin outfoldings on LV transduction of Mtmr2-null explants with FLAG-MTMR2 $(17.99 \pm 2.20 ; n=9)$ and FLAG-MTMR2 $\Delta$ PDZBD $(29.97 \pm 1.76 ; n=9)(p=0.00085) ;$ FLAG-MTMR2 $\Delta$ PDZBD $(29.97 \pm 1.76 ; n=9)$ and GFP $(76.5 \pm$ $5.0 ; n=4)(p=0.00097)$ (2 different experiments). Error bars indicate SEM. ${ }^{* *} p<0.001 ;{ }^{* * *} p<0.0001$. Scale bars: (in $\left.\boldsymbol{H}\right) \boldsymbol{A}, \boldsymbol{B}, 20 \mu \mathrm{m} ; \boldsymbol{A}^{\prime}, \boldsymbol{B}^{\prime}, 14 \mu \mathrm{m} ; \boldsymbol{G}, \boldsymbol{H}, 10 \mu \mathrm{m}$; (in $\left.\boldsymbol{C}\right) \boldsymbol{C}, 100 \mathrm{~nm}$. 
observed in vivo in Mtmr2-null nerves, Dlg1 localization at nodal-paranodal regions is not altered in Mtmr2-null cocultures (data not shown), suggesting a different turnover of this protein in vitro.

\section{Mtmr2 replacement rescues myelin outfoldings in vitro}

To demonstrate that myelin outfoldings in vitro are caused by loss of Mtmr2, we replaced FLAG-MTMR2 phosphatase in Mtmr2null explants by means of LV transduction. We used a LV encoding GFP as control. Explants were transduced at MOI 50 after neurite extension and $2 \mathrm{~d}$ before induction of myelination. Both GFP and FLAG-MTMR2 were expressed by almost all Schwann cells, suggesting that we could achieve high transduction efficiency (Fig. 2A,B). Quantitative PCR analysis performed on DNA extracted from transduced explants confirmed the integration of $\geq 30$ copies of FLAG-MTMR2-LV per cell. Sustained MTMR2 overexpression did not alter myelin structure and compaction as assessed by electron microscopy on transduced cocultures (Fig. 2C). FLAG-MTMR2 replacement in mutant explants led to up $80 \%$ reduction of fibers displaying myelin outfoldings, demonstrating that loss of Mtmr2 provokes the dysmyelination in vitro (Fig. $2 A^{\prime}, B^{\prime}, D$ ) [not transduced (NT), $75.5 \pm 2.3$; GFP, $76.5 \pm 5$; FLAG-MTMR2, $\left.10.7 \pm 5.3 ; p=8.7 \times 10^{-6}\right]$. Thus, Mtmr2-null Schwann cell/DRG neuron cocultures represent a useful model to explore the function of Mtmr2 during myelination.

\section{Loss of Mtmr2/Dlg1 interaction contributes to myelin outfolding formation}

We then exploited Mtmr2-null Schwann cell/DRG neuron cocultures to assess whether loss of Mtmr2/Dlg1 interaction contributes to the dysmyelinating phenotype. We transduced Mtmr2null explants with LV encoding either FLAG-MTMR2 or FLAGMTMR2 $\triangle$ PDZBD, a construct lacking the postsynaptic density95/Discs large/zona occludens-1 (PDZ)-binding domain and thus unable to bind Dlg1 (Fig. 2E). Using two different transduction protocols (MOI 50 and 25), we observed almost a full rescue with both constructs. Since in our hypothesis Dlg1 might transport and/or cluster Mtmr2 at sites of membrane remodeling, we speculated that when expressed at high levels Mtmr2 might diffuse and function despite the absence of interaction with Dlg1. We therefore titrated down the MOI of the vector. At MOI 12.5, FLAG-MTMR2 $\triangle$ PDZBD LV rescued myelin outfoldings significantly less effectively than FLAG-MTMR2 (Fig. 2G-I) (GFP, $76.5 \pm$ 5; FLAG-MTMR2 $\Delta$ PDZBD, $29.97 \pm 1.76$; $p=0.00097$; FLAG-MTMR2, $17.99 \pm 2.20$; FLAG-MTMR2 $\triangle$ PDZBD, $29.97 \pm 1.76 ; p=0.00085)$, despite similar extent of integration of the two LV (16.3 copies for FLAG-MTMR2-LV and 16.4 for FLAG-MTMR2 $\triangle$ PDZBD-LV) and similar expression of the two transgenes (Fig. 2F). This finding suggests that loss of Mtmr2/ Dlg1 interaction contributes to the dysmyelinating phenotype.

\section{Dlg1 interacts with kif13B and Sec8 in Schwann cells in the nerve}

We then hypothesized that loss of Mtmr2/Dlg1 interaction contributes to myelin outfoldings mainly because Dlg1 might assemble multiprotein complexes that regulate polarized vesicle trafficking and coordinate membrane formation and its control. We thus searched for Dlg1 interactors in a rat nerve cDNA library by yeast two-hybrid screening analysis using several portions of the Dlg1 cDNA as bait. Among others, we focused on two putative binding partners, previously involved in polarized vesicle trafficking in other cells: kif13B, a plus end kinesin motor protein of the kinesin 3 family (Hanada et al., 2000), and the Sec8 exocyst component (Hsu et al., 2004). We identified Kif13B by using the Dlg1/SH3-HOOK-GUK (Src homology domain 3-guanylate kinase-like) cDNA fragment as bait and Sec 8 by using the Dlg1/ PDZ2 +3 cDNA. The latter bait also demonstrated interaction with Mtmr2 in the same screening, thus confirming once more the previously reported Mtmr2/Dlg1 complex (Bolino et al., 2004).

We further assessed the kif13B/Dlg1 interaction in the nerve by performing GST pull-down assays from rat nerve lysates at P11. Since myelin outfoldings arise $~ 3-4$ weeks after birth, we speculated that the molecular interactions relevant to the phenotype would be most likely detected before that period. Previous studies have already shown that the GUK domain of Dlg1 mediates binding with the MAGUK binding stalk (MBS) domain of kif13B (Hanada et al., 2000). Thus, we used either recombinant GST-Dlg1/GUK or GST-kif13B/MBS fusion proteins to identify this interaction in $\mathrm{P} 11$ rat nerve lysate. We detected endogenous kif13B and Dlg1 by Western blot analysis (Fig. 3A-E), respectively. Moreover, we identified kif13B and Dlg1 using GSTkif13B/MBS in mass spectrometric analysis (Shevchenko et al., 1996) (supplemental Table 1, available at www.jneurosci.org as supplemental material), providing additional evidence that the kif13B/Dlg1 interaction occurs endogenously in the nerve. Since kif13B shares homology with KIF1A, which exists as a monomer in solution, it is unlikely that kif13B may dimerize in vivo (Okada et al., 1995). However, Dlg1 can dimerize through its PDZ or L27 domains as already demonstrated in postsynaptic density (PSD) regions for Dlg1 and also for PSD-95, another PDZ domaincontaining scaffolding protein (Kim and Sheng, 2004; Nakagawa et al., 2004).

By performing immunohistochemistry in vivo, we assessed that kif13B expression is mainly enriched in myelin-forming and non-myelin-forming Schwann cells in nerve (Fig. $3 F-H$ ). In myelinated fibers, kif13B was also found at the abaxonal Schwann cell cytoplasm in structures resembling Cajal bands, with an enrichment in microvilli, paranodes, and Schmidt-Lanterman incisures (SLIs) (Fig. 3I,J). Moreover, in Mtmr2-null nerves, kif13B levels and localization were not altered (Fig. $3 \mathrm{~K}-\mathrm{O}$ ).

We further confirmed interaction between Dlg1 and Sec 8 by performing coimmunoprecipitation from P11 mouse nerve lysates (Fig. 4A,B). The abundant expression of both Dlg1 and Sec8 in vivo allowed us to detect both proteins at the endogenous level. In the nerve, Sec8 was mainly detected in the cytosol and at the plasma membrane of myelin-forming Schwann cells (Fig. $4 D$ $F)$. In teased-fiber analysis, $\operatorname{Sec} 8$ was detected in microvilli and SLIs (Fig. 4G), paranodes (Fig. $4 H$ ), and, most abundantly, in the Golgi (Fig. 4I). Finally, Sec8 levels and localization were not altered in Mtmr2-null nerves (Fig. 4C,J,K).

\section{kif13B pulls down Dlg1, Sec8, and Mtmr2, which also colocalize in vivo}

We provided evidence that Mtmr2, kif13B, and Sec 8 are all binding partners of Dlg1 in the nerve. All these interactions likely occur in Schwann cells in the nerve since (1) yeast two-hybrid screening analysis was performed using a cDNA library enriched for Schwann cell mRNAs; (2) we had previously demonstrated in vivo that Dlg1 is localized in myelin-forming Schwann cells and not in axons (Bolis et al., 2005); and (3) here, we report that both kif13B and Sec8 expression is mainly enriched in myelin-forming Schwann cells. To better characterize these complexes, we performed pull-down assays by using a recombinant GST-Mtmr2, since the phosphatase is expressed at very low levels in the nerve. 
A

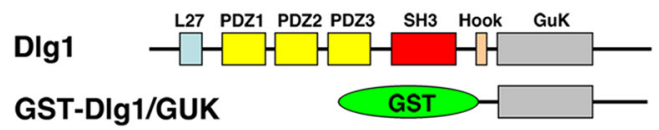

B

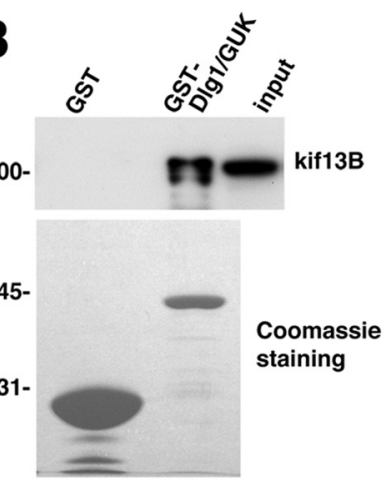

C

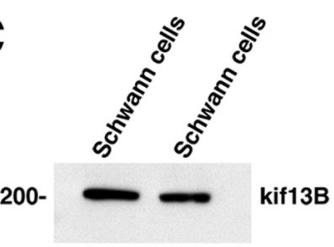

D
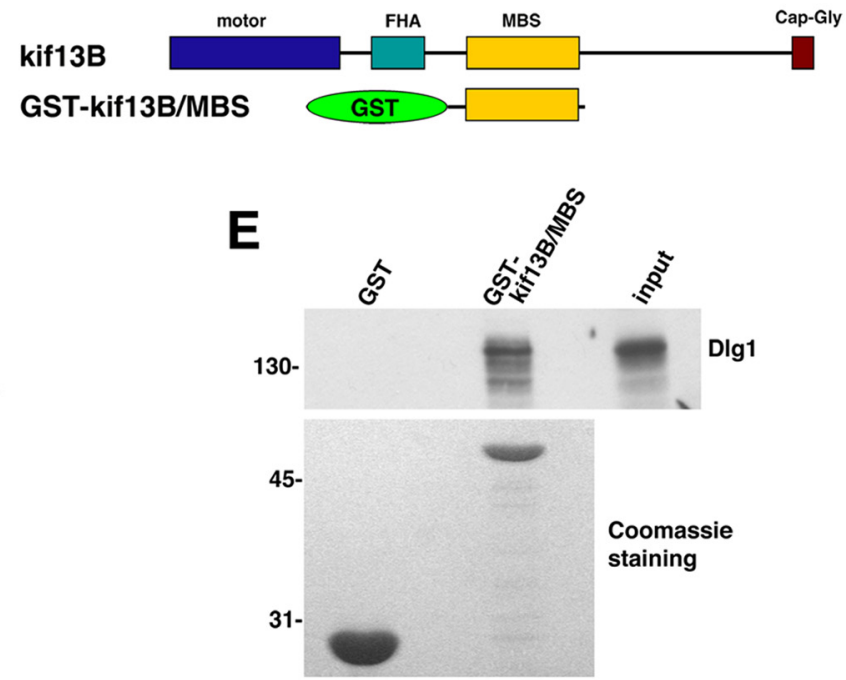
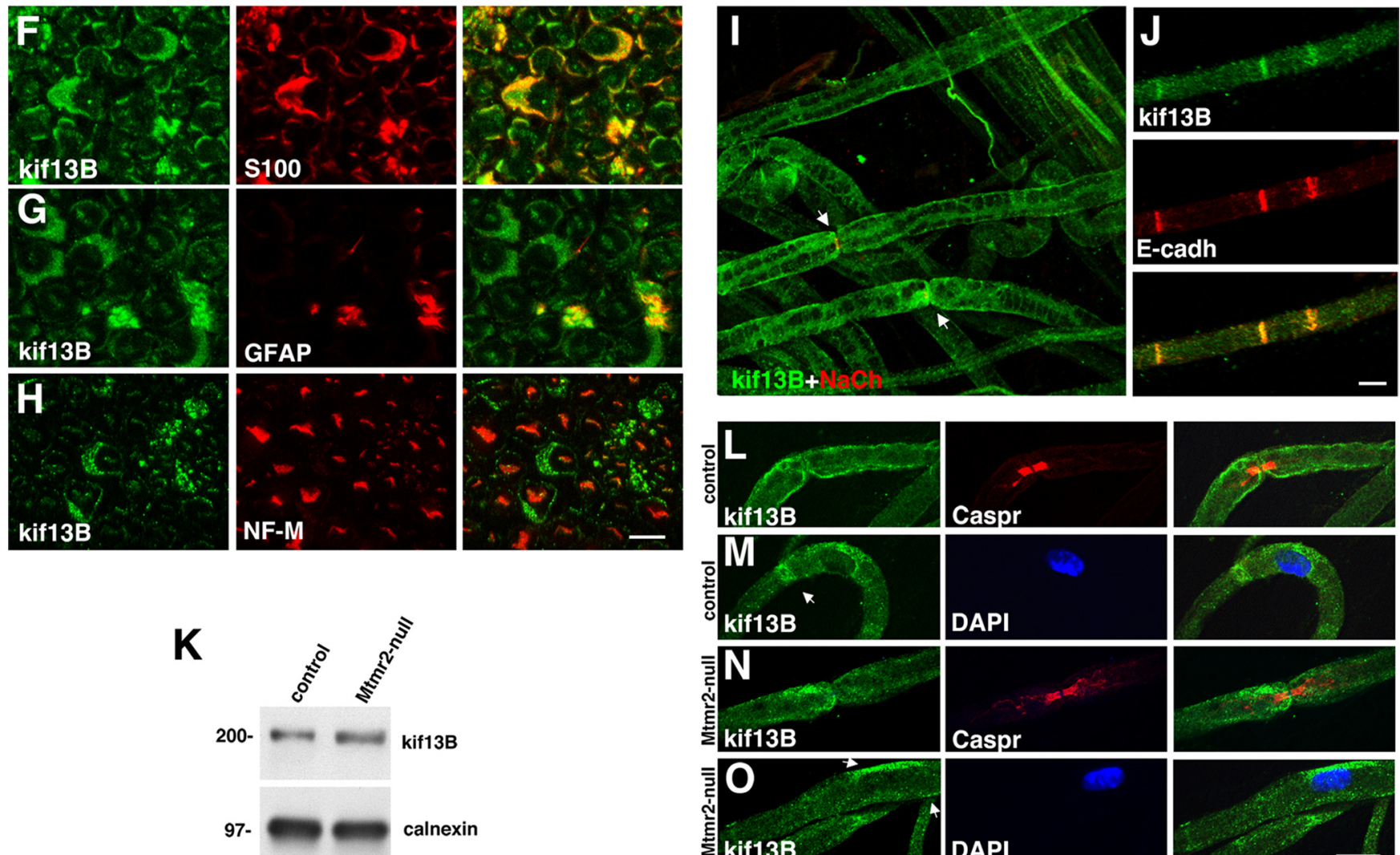
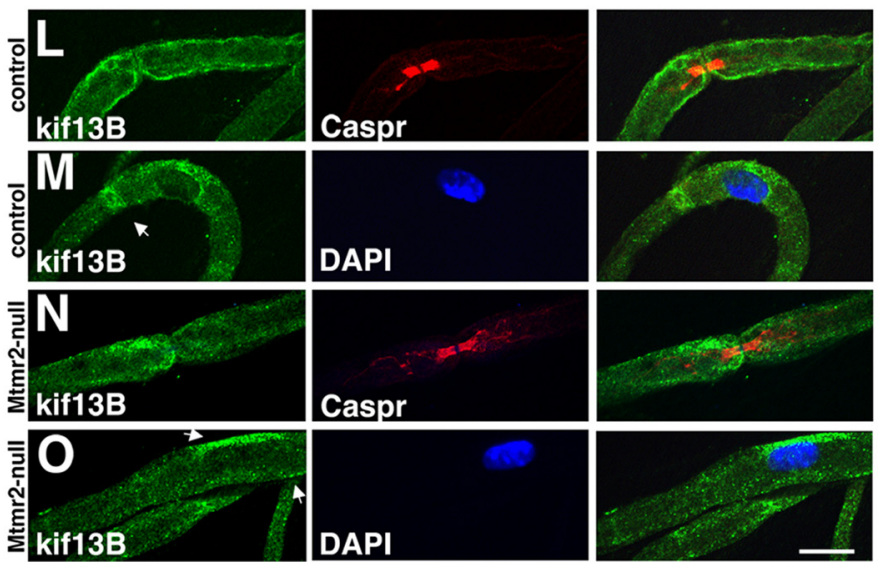

Figure 3. Dlg1 interacts with kif13B. A-E, Dlg1/kif13B interaction was further demonstrated by performing pull-down assays on P11 rat nerve lysates using GST-Dlg1/GUK and GST-kif13B/MBS, in which the input is the nerve lysate. C, Western blot analysis confirmed kif13B expression in isolated rat Schwann cells. $F, G$, In vivo, in transverse rat nerves, kif13B is expressed in the cytosol of both myelin-forming and in non-myelin-forming Schwann cells, colocalizing with the cytosolic marker S100. G, GFAP staining identified non-myelin-forming Schwann cells. $\boldsymbol{H}$, kif13B is also expressed in axons, although less abundantly. II, In teased-fiber analysis, kif13B expression was detected in internodal cytoplasmic channels and microvilli-paranodes as indicated by arrows (node). $J$, kif13B was also detected in SLIs. $\mathbf{L}-\mathbf{O}$, Immunohistochemistry performed on $M$ tmr2-null fibers showed normal kif13B localization. The arrows indicate SLIs in $\boldsymbol{M}$, and, in $\mathbf{O}$, perinuclear staining. $\boldsymbol{K}$, kif13B expression level in Mtmr2-null nerves is not altered as shown by Western blot analysis. Sciatic nerves from 2- to 3-month-old animals were used. Scale bars, $10 \mu \mathrm{m}$.

By Western blot analysis, we detected Dlg1 and Sec8 but not kif13B in both P11 rat nerve as well as in rat Schwann cell lysates (Fig. 5A,B) (data not shown). We also performed pull-down assays from P11 rat nerve and Schwann cell lysates using recombinant GST-kif13B/MBS, and we repeatedly detected Dlg1, Sec8, and Mtmr2 (Fig. 5C,D).

We identified Sec8 and Mtmr2 as binding partners of Dlg1 using the Dlg1/PDZ2+3 cDNA fragment as bait. To assess whether Sec8 and Mtmr2 bind to distinct PDZ domains of Dlg1, we transfected COS7 cells with either myc-Mtmr2 or GFP-Sec8. We performed pull-down assays from lysates of transfected cells using recombinant proteins of different domains of Dlg1 fused to GST (supplemental Fig. 1, available at www.jneurosci.org as supplemental material). Mtmr2 and Sec8 displayed preferential 
binding to PDZ2 and PDZ1+2, respectively, suggesting that the two proteins may bind to distinct Dlg1 domains. Interestingly, in the brain, Sec 8 also binds to PDZ1+2 of SAP102, another PDZ domain-containing scaffold (Sans et al., 2003).

In conclusion, we demonstrated that, in Schwann cells, kif13B pulls down Dlg1, which in turn binds to Sec8 and Mtmr2. Since Mtmr2 and Sec8 may bind to distinct PDZ domains of Dlg1, it is likely that Dlg1, Sec8, and Mtmr2 belong to the same complex.

We then investigated whether these molecules also colocalize in vivo in teasedfiber analysis. At P11 when molecular interactions were detected, kif13B, Sec8, and Dlg1 were found in the same cytoplasmic regions of noncompact myelin such as microvilli, paranodes, and incisures in which cytosolic Mtmr2 has also been detected (Previtali et al., 2003b; Bolino et al., 2004).

Dlg1 is differentially localized during development with a nodal localization at earlier stages starting from P5. At later stages and in adult fibers, Dlg1 localizes at paranodes and at SLIs. We did not observe any change in kif13B and Sec8 localization during development. Moreover, kif13B staining always appears cytosolic, whereas Dlg1 and Sec8 are also enriched at the abaxonal Schwann cell membrane of microvilli and paranodes in fibers from 3-month-old animals, consistent with the role of both Dlg1 and Sec8 in polarized vesicle tethering at the plasma membranes (supplemental Fig. 2, available at www. jneurosci.org as supplemental material). Finally, kif13B, Dlg1, and Sec8 expression levels are upregulated during myelination (supplemental Fig. 2, available at www. jneurosci.org as supplemental material).

\section{kif13B transports Dlg1 at sites of membrane remodeling}

kif13B transports Dlg1 to the tip of cell projections in MDCK cells (Asaba et al., 2003), and, in oligodendrocytes, Sec8 promotes membrane formation through interaction with the multidomain CASK scaffold (Anitei et al., 2006). We therefore hypothesized that, in Schwann cells, kif13B might transport Dlg1 to sites of membrane remodeling. Here, interaction of Dlg1 with Sec8 promotes membrane addition, whereas with Mtmr2, negatively regulates membrane formation. These complexes might coordinate a homeostatic control of myelination. Sec8 and Mtmr2 might be either transported by kif13B as a complex with Dlg1 or recruited and clustered by the Dlg1 scaffold at the

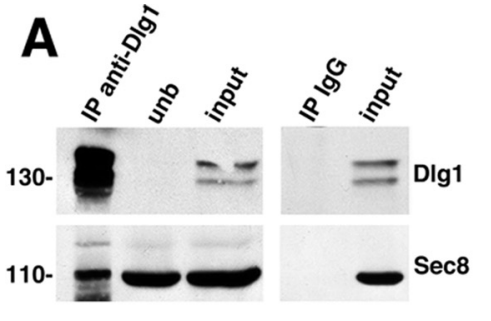

B
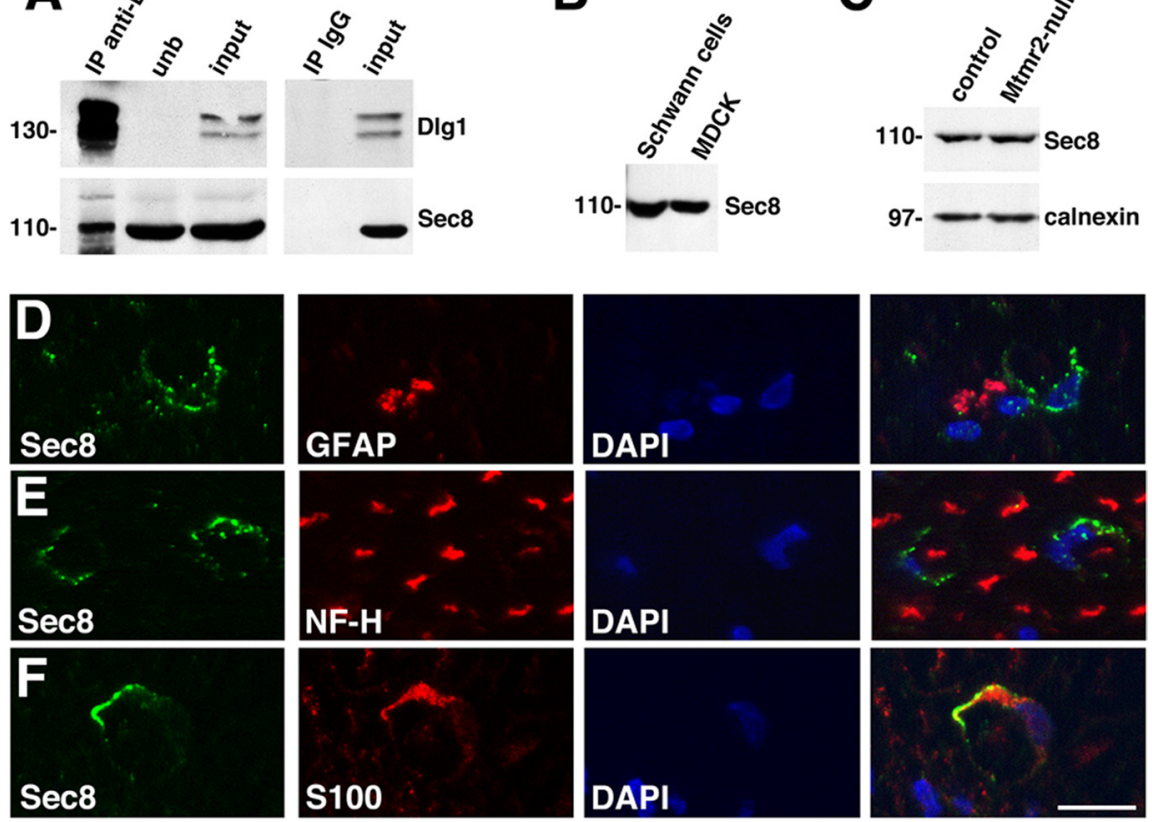

G

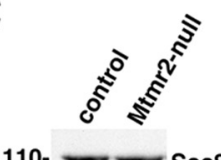

97- - calnexin
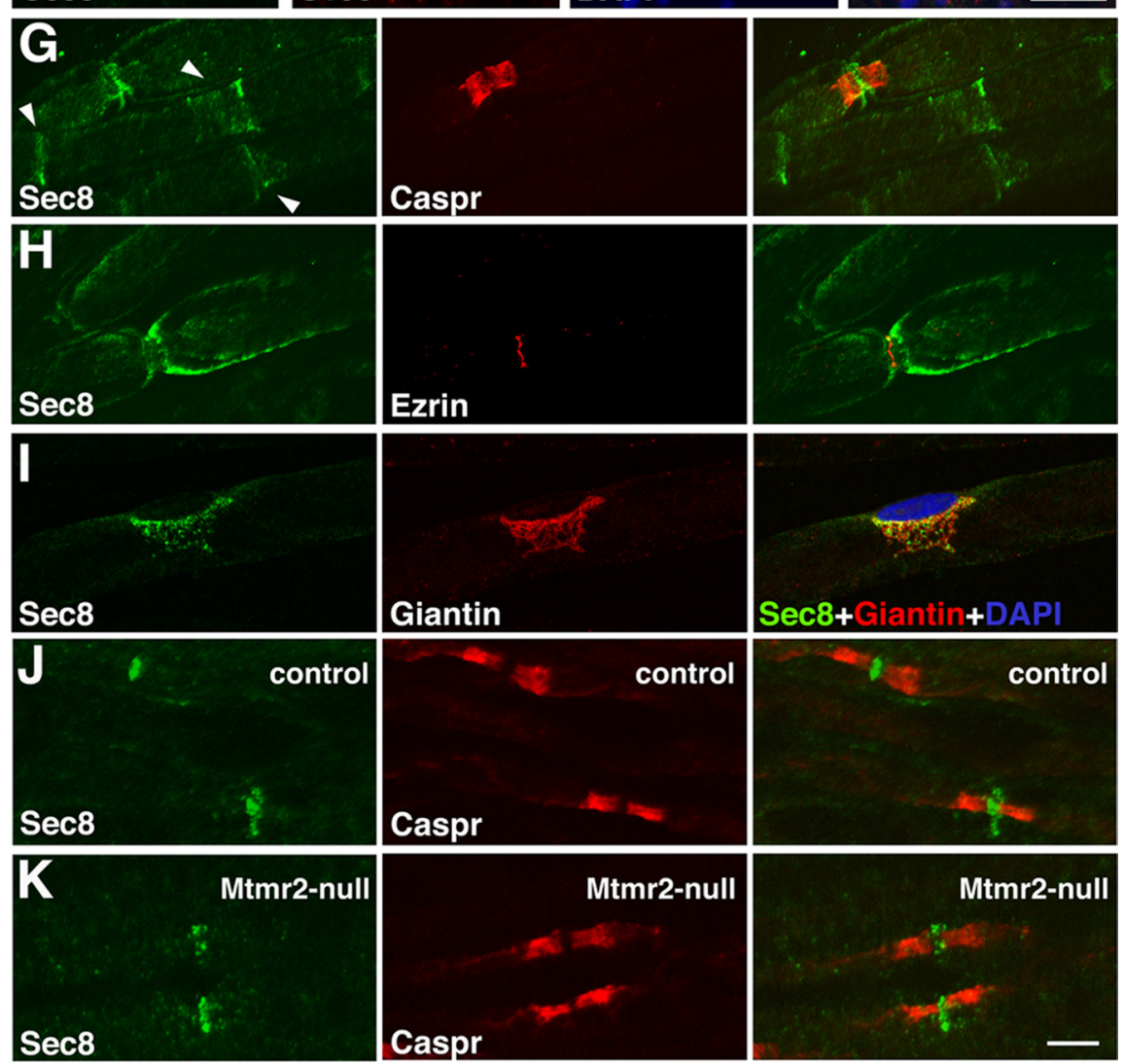

Sec8+Giantin+DAPI
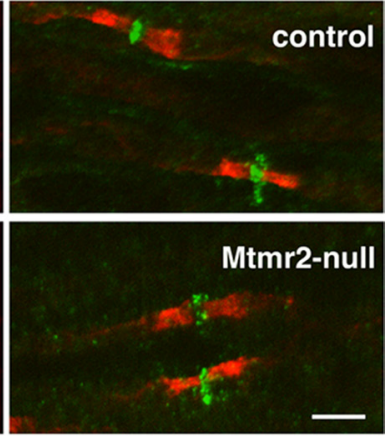

Figure 4. Dlg1 interacts with Sec8. $\boldsymbol{A}$, Coimmunoprecipitation of Dlg1 and Sec8 from mouse nerves. Input is the lysate; "unb" is the unbound fraction of the lysate after immunoprecipitation. $\boldsymbol{B}$, Western blot analysis showing Sec8 expression in isolated rat Schwann cells, with MDCK cell lysate as control. C, Western blot analysis confirmed that Sec8 expression level in Mtmr2-null nerve lysate is not altered. $\mathbf{D}-\boldsymbol{F}$, Immunohistochemistry on transverse rat nerve sections revealed a Sec8 punctate staining in the cytoplasm of myelin-forming Schwann cells, S100 positive, but not in non-myelin-forming Schwann cells, GFAP positive, nor in axons. $\boldsymbol{G}, \boldsymbol{H}$, Immunohistochemistry on rat teased nerves showed Sec8 expression in SLIs (arrowheads) and in the microvilliparanodes. Caspr is a marker of paranodes in the axon. I, A strong Sec8 perinuclear expression was also detected as shown by colocalization with the Golgi marker Giantin. $\boldsymbol{J}, \boldsymbol{K}$, Immunohistochemistry on Mtmr2-null mouse nerves, showing that Sec8 expression at microvilli is not altered with respect to control fibers. Sciatic nerves from 2-to 3-month-old animals were used. Scale bars: (in $\boldsymbol{F}$ ) $\boldsymbol{D}-\boldsymbol{F}, 5 \mu \mathrm{m}$; (in $\boldsymbol{K}) \mathbf{G}-\boldsymbol{I}, 10 \mu \mathrm{m} ; \boldsymbol{J}, \boldsymbol{K}, 5 \mu \mathrm{m}$. 
A

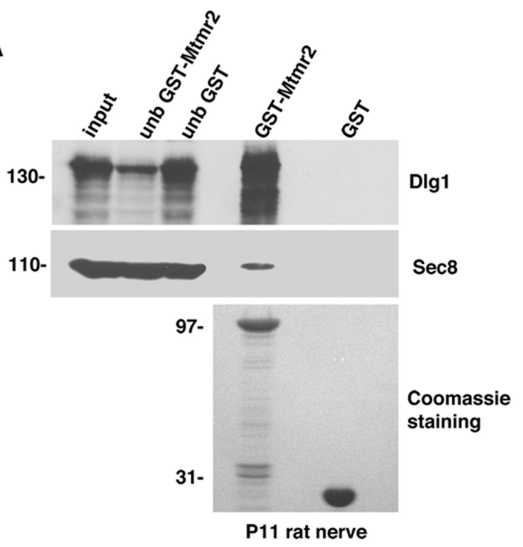

C

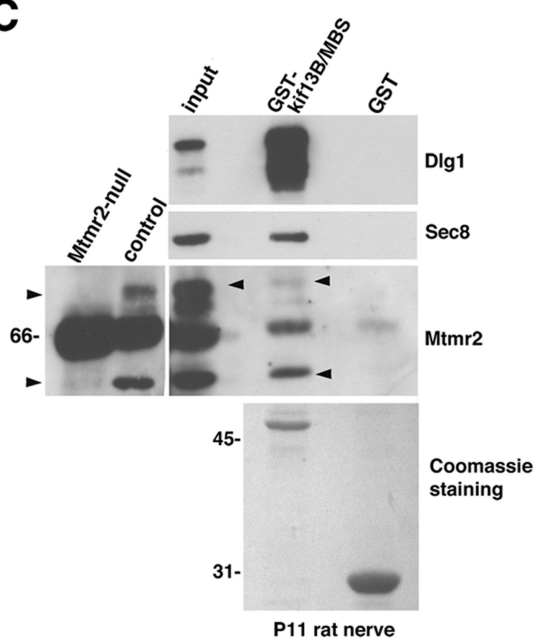

B

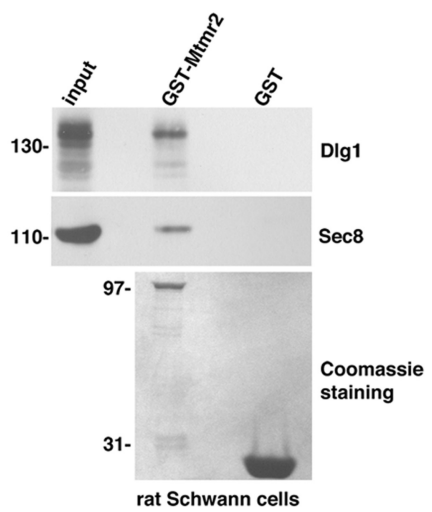

D

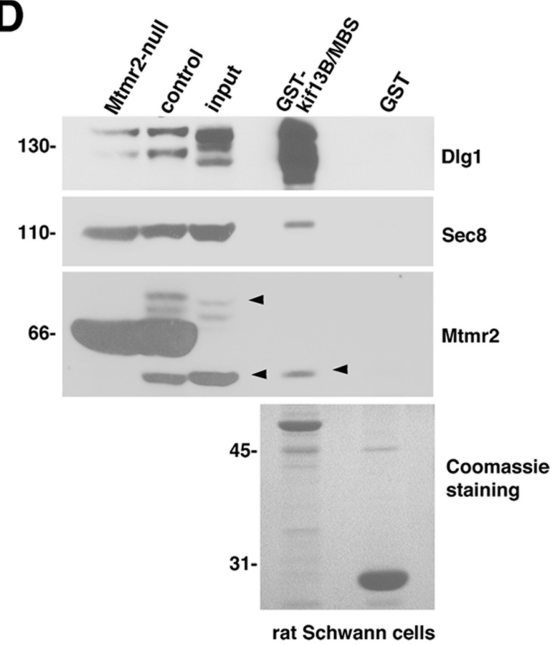

Figure 5. GTS-kif13B/MBS pulls down Dlg1, Sec8, and Mtmr2 in both nerve and isolated Schwann cells. $\boldsymbol{A}$, Pull-down assay using GST-Mtmr2 on P11 rat nerves. Sec 8 and Dlg1 were revealed by Western blot analysis. $\boldsymbol{B}$, The same experiment was performed from isolated rat $S$ chwann cells lysate to confirm that these interactions likely occur in Schwann cells in the nerve. $C, D$, Pull-down assays using GST-kif13B/MBS from P11 rat nerve and isolated rat Schwann cell lysates, in which Sec8, Dlg1, and Mtmr2 were detected. Mtmr2-null and wild-type mouse nerve lysates were also used as control to test the specificity of the anti-Mtmr2 antibody used. Two bands of $\sim 70$ and $60 \mathrm{kDa}$ disappear in the Mtmr2-null nerve lysate, suggesting that this antibody recognizes Mtmr2. Input is the lysate, and "unb" is the unbound fraction after pull down.

abaxonal membranes. Excessive myelin in Mtmr2-null nerves could be attributable to loss of negative control on Sec8-mediated membrane formation.

To confirm this model, we perturbed the transport mediated by kif13B using dominant-negative forms of the kinesin. We transduced at MOI 50 Schwann cell/DRG neuron cocultures with a LV encoding myc-kif13B $\Delta \mathrm{MD}$, a kif13B lacking the $\mathrm{N}$ terminus motor domain, which is still able to interact with Dlg1 (Fig. $6 A, B, D, E)$. This truncated form of kif13B has been used previously to demonstrate that kif13B transports Dlg1 to the tip of MDCK cell projections (Asaba et al., 2003) and $\operatorname{PtdIns}(3,4,5) \mathrm{P}_{3}$ (also known as $\mathrm{PIP}_{3}$ ) to the tips of growing neurons through a $\mathrm{PIP}_{3} \mathrm{BP}$ ( $\mathrm{PIP}_{3}$-binding protein) complex (Horiguchi et al., 2006). Following on the hypothesis that, in Schwann cells, kif13B transports Dlg1 at nodal-paranodal regions, first we quantified Dlg1 staining in myelinated fibers. Accordingly, we found that Dlg1 localization was significantly altered (Fig. $6 F-J$ ), whereas cultures transduced with GFP-LV or other LV encoding myc-tag fusion proteins have normal Dlg1 localization (data not shown). We obtained the same result using a different dominant-negative construct, myc-kif13B/MBS, which encodes only the kif13B domain responsible for Dlg1 binding (Fig. 6A, C). Surprisingly, we also observed a significant reduction in the amount of MBP-positive myelinated fibers of explants transduced with this myckif13B/MBS LV (Fig. $6 K-N$ ), suggesting that the loss of kif13B-mediated Dlg1 transport may impair active myelination.

kif13B, Dlg1, and Sec8 are involved in Schwann cell myelination

To further assess the role of kif13B/Dlg1, we explored whether their loss of function affects myelination. We selected a kif $13 B$ shRNA LV, which can reduce kif13B expression up to $86 \%$ in isolated rat Schwann cells (Fig. 7A). Interestingly, the depletion of kif13B also resulted in a decrease of both Dlg1 (up to 41\%) and Sec8 (up to 59\%) expression levels (Fig. 7A) (at least three independent experiments). Using this kif13B shRNA LV, we then transduced Schwann cell/DRG neuron cocultures, and we observed that Schwann cells were preferentially transduced (supplemental Fig. 3, available at www.jneurosci. org as supplemental material). After induction of myelination, transduced explants displayed a significant reduction of MBP-positive myelinated fibers (supplemental Fig. 4, available at www. jneurosci.org as supplemental material). To rule out that the decrease in myelination was a consequence of a decrease of the Schwann cell number, we also established dissociated Schwann cells/DRG neurons in which Schwann cell nuclei can be more easily counted. In these cultures, both Schwann cells and neurons were transduced by shRNA LV (supplemental Fig. 3, available at www.jneurosci.org as supplemental material). We thus confirmed that depletion of kif13B impairs myelination (Fig. 7B,D-F). Finally, Schwann cell/DRG neuron cocultures transduced with a Dlg1 shRNA LV displayed a similar decrease in myelination, thus supporting a role of the kif13B/Dlg1 complex in myelination (Fig. 7C, $G-I$ ).

Since (1) loss of kif13B perturbs Dlg1 localization, (2) loss of both kif13B and Dlg1 expression impairs active myelination, and (3) Dlg1 also interacts with Sec8, which in oligodendrocytes promotes differentiation and membrane formation (Anitei et al., 2006), we assumed that a decrease in Sec8 function might contribute to the observed hypomyelination. To provide evidence for this hypothesis, we selected a $\operatorname{Sec} 8$ shRNA LV, which in transduced isolated rat Schwann cells was able to deplete Sec8 expression up to $68 \%$ (supplemental Fig. 5, available at www.jneurosci. org as supplemental material). Both DRG explants and dissociated Schwann cell/DRG neuron cocultures transduced with this Sec8 shRNA LV were significantly less myelinated compared with control not transduced cultures (supplemental Fig. 5, available at www.jneurosci.org as supplemental material; Fig. 8A-D). As already observed in oligodendrocytes (Anitei et al., 2006), Sec8 shRNA transduced cocultures displayed a reduction in Schwann cell number. Nevertheless, the decrease in myelination (up to 
A

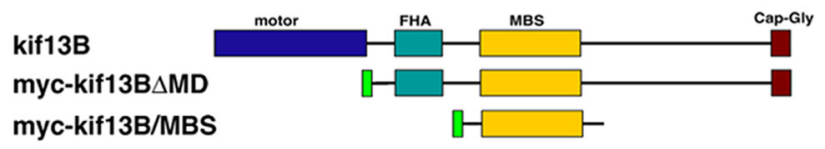

B

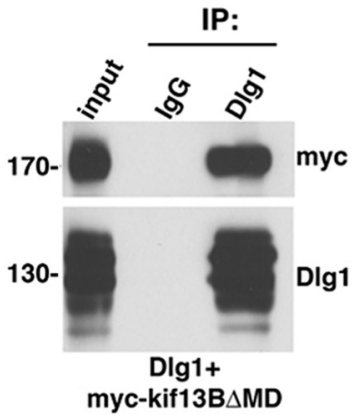

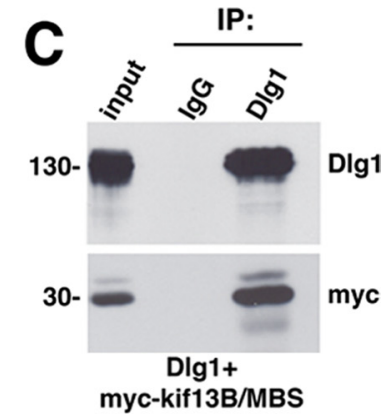
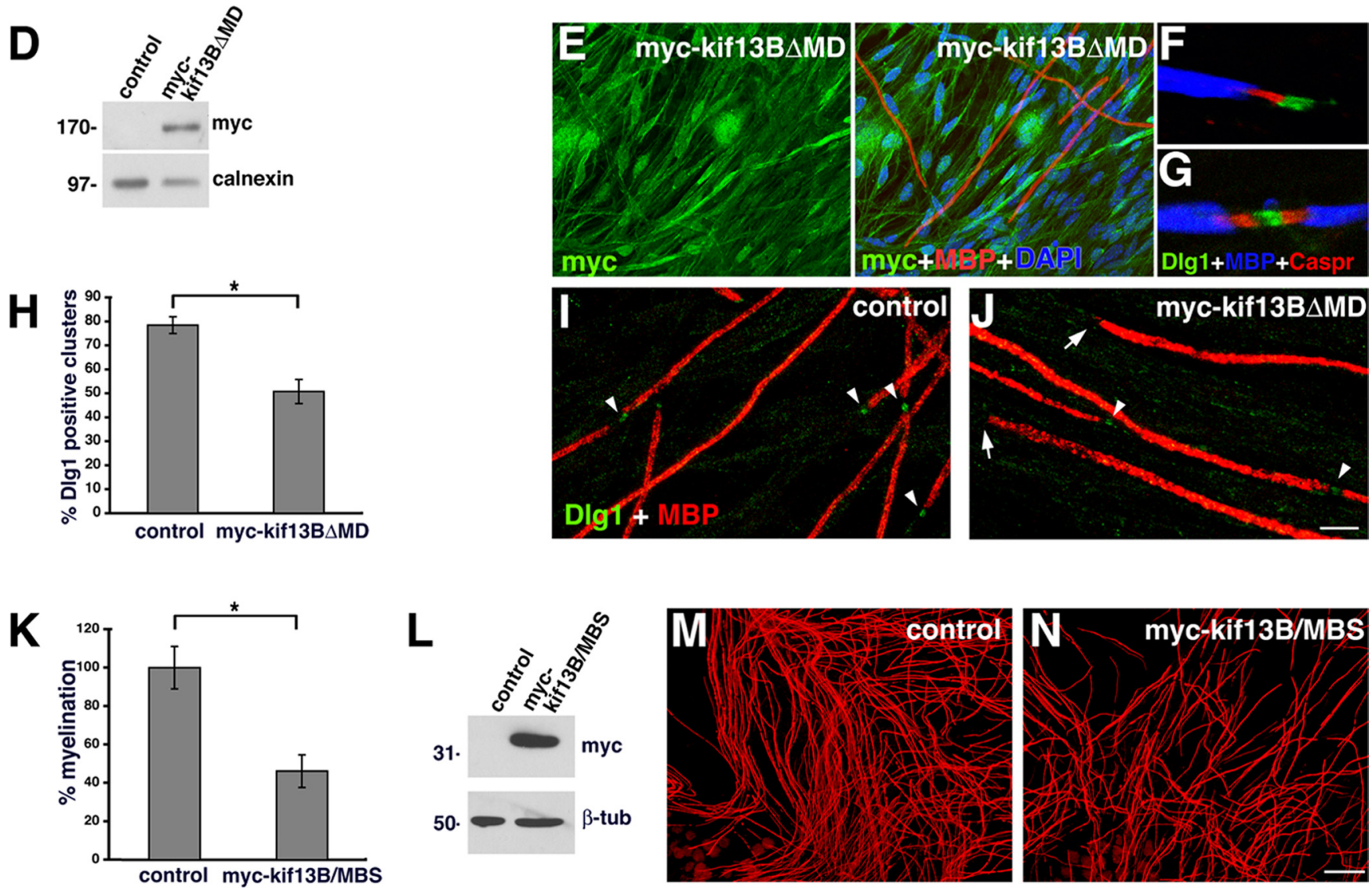

Figure 6. kif13B transports Dlg1 in Schwann cells. $A$, Dominant-negative constructs of kif13B used in myelin-forming cocultures. $B, C$, Coimmunoprecipitation in COS7 cells between overexpressed myc-kif13B $\triangle M D$ and Dlg1 and between overexpressed myc-kif13B/MBS and Dlg1.D, Western blot analysis on lysates from myelin-forming cocultures transduced with myc-kif13B $\Delta M D$ LV, in which control is the GFP LV. $E$, myc-kif13B $\Delta M D$ LV is expressed in almost all cells with high copy number integration. $F, G$, Dlg1 localization in myelin-forming cocultures appears at microvilli similar to what has been observed in immature nerves in vivo during postnatal development (see also supplemental Fig. $2 \mathrm{~A}-\mathrm{C}$, available at www.jneurosci.org as supplemental material). $\boldsymbol{H}-\boldsymbol{J}$, Quantification of Dlg1-positive clusters in myelinated fibers stained with MBP on myc-kif13B $\Delta$ MD overexpression (myc-kif13B $\Delta M D, 50.74 \pm 5.04, n=6 ;$ GFP, 78.40 $\pm 3.49, n=7 ; p=0.00146$; 2 experiments). L, Western blot analysis on lysates from myelin-forming cocultures transduced with myc-kif13B/MBS LV, in which control is GFP LV. $\boldsymbol{K}$-N, Quantification of MBP-positive fibers in explants overexpressing myc-kif13B/MBS (myc-kif13B/MBS, $46.17 \pm 8.50, n=6 ; \mathrm{GFP}, 100 \pm 11.07, n=4 ; p=0.00997$ ). Error bars indicate SEM. ${ }^{*} p<0.05$. Scale bars: (in $J$ ) $\boldsymbol{I}, \boldsymbol{J}, 10 \mu \mathrm{m} ; \boldsymbol{E}, 30$ $\mu \mathrm{m} ; \boldsymbol{F}, \mathbf{G}, 2 \mu \mathrm{m}$; (in $N) \boldsymbol{M}, \boldsymbol{N}, 100 \mu \mathrm{m}$.

$80 \%$ ) in treated culture is not proportional to the decrease in the Schwann cell number (up to $20 \%$ ).

Our results indicate that kif13B transports Dlg1 to sites of membrane remodeling in Schwann cells and that interaction of Dlg1 with the Sec8 exocyst component promotes membrane formation during active myelination.

Reduced Sec8 expression in Schwann cells rescues myelin outfoldings

We previously demonstrated that Dlg1 interacts with Mtmr2 in Schwann cells in vivo (Bolino et al., 2004). Loss of Mtmr2mediated negative control on membrane formation might pro- voke myelin outfoldings, redundant folds of excessive myelin (Bolino et al., 2004). We therefore hypothesized that Dlg1, by serving as a scaffold platform, might coordinate Sec8 and Mtmr2mediated opposite functions, which have to be properly balanced to achieve membrane homeostasis. To test this hypothesis, we tried to rescue myelin outfoldings in Mtmr2-null cocultures by interfering with Sec8 expression. We thus infected Mtmr2-null explants with either Sec8 shRNA or control shRNA, and we quantified MBP-positive fibers displaying myelin outfoldings (supplemental Fig. 5, available at www.jneurosci.org as supplemental material). We found that the percentage of myelin outfoldings in explants treated with $\operatorname{Sec} 8$ shRNA was significantly reduced com- 

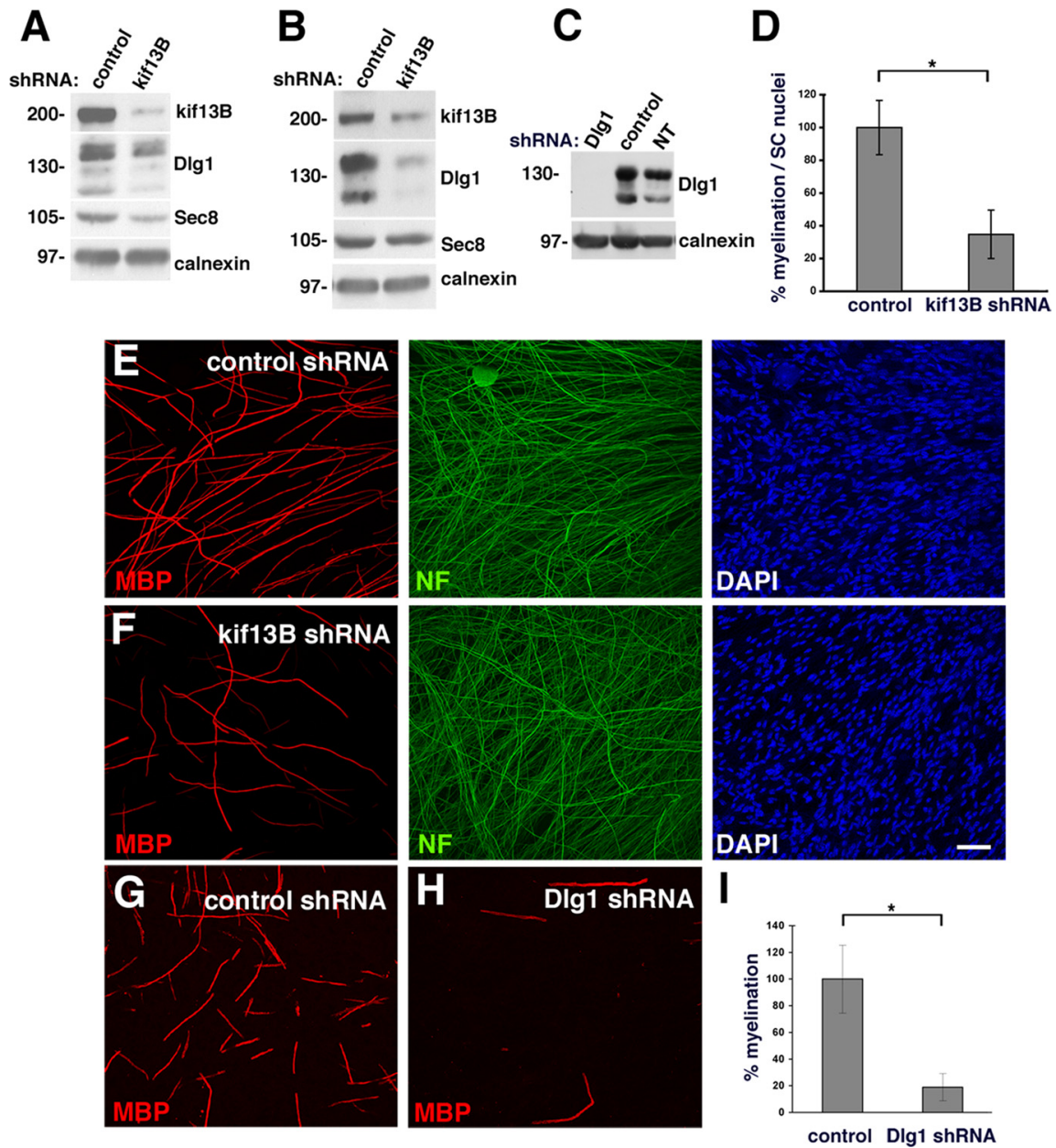

Figure 7. Loss of kif13B and Dlg1 in myelin-forming cocultures impairs active myelination. $A$, kif13B shRNA LV transduction of isolated rat Schwann cells revealed a decrease of kif13B, Dlg1, and Sec8 expression. Three independent experiments were performed. $\boldsymbol{B}$, kif13B shRNA LV transduction of dissociated Schwann cell/DRG neuron cocultures also revealed a decrease of kif13B, Dlg1, and Sec8 expression. C, Downregulation of Dlg1 expression on Dlg1 shRNA LV transduction of isolated rat Schwann cells. $D-F$, In dissociated cultures transduced with kif13B shRNA LV, myelination, as the ratio between the number of MBP-positive segments and number of Schwann cell nuclei, was significantly decreased with respect to control shRNA (kif13B shRNA, $34.80 \pm$ $14.73, n=12$; control shRNA, $100 \pm 16.60, n=16 ; p=0.00779 ; 2$ experiments). $\mathbf{G}-\mathbf{I}$, The amount of MBP-positive segments is also decreased in Schwann cell/DRG neuron cocultures on Dlg1 shRNA transduction (Dlg1 shRNA, $19 \pm 10.2, n=7$; control shRNA, $100 \pm 25.5, n=7 ; p=0.0107)$. Error bars indicate SEM. ${ }^{*} p<0.05$. Scale bar, $50 \mu \mathrm{m}$.

pared with explants infected with a control shRNA (supplemental Fig. 5, available at www.jneurosci.org as supplemental material).

The overall number of MBP-positive segments was also decreased in explants treated with Sec8 shRNA as loss of Sec8 impairs active myelination (data not shown). To rule out the possibility that the observed rescue was attributable to the decrease in myelination, we performed a titration experiment on dissociated Schwann cell/DRG neuron cocultures to determine the highest dose of $\operatorname{Sec} 8$ shRNA LV able to rescue myelin outfoldings without impairing active myelination (Fig. $8 E-G$ ). Using this amount of Sec8 shRNA LV, we transduced Mtmr2-null dissociated cocultures and we found that myelin outfoldings were significantly rescued without affecting myelination (Fig. $8 \mathrm{H}-\mathrm{J}$ ).

These results suggest that, when the Mtmr2-mediated negative control of membrane formation is lost, unbalanced Sec8mediated membrane addition leads to excessive myelin and myelin outfoldings.

\section{Discussion}

The cell biological hallmark of myelination is membrane biosynthesis, the synthesis and transport of bulk of proteins to produce a unique extension of the glial cell plasma membrane that wraps around the axon (Trapp et al., 2004). However, molecular interactions responsible for sorting and site-specific docking, tethering, and fusion of vesicles have not yet been identified (Simons and Trotter, 2007). Here, we provide evidence for the first molecular mechanism that titrates membrane formation during Schwann cell myelination.

Loss of the MTMR2 phospholipid phosphatase in humans and mice causes CMT4B1 demyelinating neuropathy with myelin outfoldings, excessive redundant myelin in the nerve (Bolino et al., 2000, 2004; Bolis et al., 2005). We previously suggested that Mtmr2 might coordinate membrane formation during Schwann cell myelination in a complex with the Dlg1 scaffolding protein. To explore Mtmr2/ Dlg1 function during myelination, we established myelin-forming Schwann cell/ DRG neuron cocultures from Mtmr2-null mice in which myelin outfoldings were reproduced and almost completely rescued by Mtmr2 replacement. To our knowledge, this is the first example of abnormal myelination, which is morphologically reproduced in vitro. Taking advantage of this model, we thus demonstrated that loss of Mtmr2/Dlg1 interaction contributes to myelin outfoldings. A MTMR2 that is not able to bind to Dlg1, MTMR2 $\triangle \mathrm{PDZBD}$, rescues myelin outfoldings less efficiently than wild-type MTMR2. We therefore hypothesized that Dlg1 clusters MTMR2 to sites of membrane remodeling where endosomal trafficking is needed to control membrane formation during postnatal development. To investigate the role of Dlg1 in Schwann cells, we searched for Dlg1 interactions. We found that Dlg1 interacts with the kif13B kinesin, and the Sec8 exocyst complex component, both involved in polarized vesicle trafficking (Hanada et al., 2000; Asaba et al., 2003; Hsu et al., 2004; Horiguchi et al., 2006). Kif13B kinesin is a plus end motor protein that in neurons transports $\mathrm{PIP}_{3}$-containing vesicle along microtubules, thus promoting neuronal polarity (Horiguchi et al., 2006). The exocyst is a complex of eight proteins in mammals involved in the posttranslational regulation of protein synthesis and polarized membrane domain formation, which is often mediated by multidomain scaffold interactions (Zhang et al., 2001; Inoue et al., 2003, 2006; Hsu et al., 2004; Anitei et al., 2006; Gerges et al., 2006; Elias and Nicoll, 2007; Gorczyca et al., 2007).

Although Dlg1/kif13B and Dlg1/Sec8 interactions have been demonstrated in other cells (Asaba et al., 2003; Inoue et al., 2006; Yamada et al., 2007), we pulled down Dlg1, Sec8, and Mtmr2 using GST-kif13B/MBS from both isolated Schwann cells and 
nerve lysates. This finding prompted us to investigate the physiological relevance of these interactions during myelination.

By interfering with the kif13Bmediated transport in myelin-forming cocultures, we found that Dlg1 was not properly localized at sites of membrane remodeling and, more intriguing, that myelination was also decreased. We further confirmed this finding by performing shRNA-mediated depletion of kif13B and Dlg1 on myelin-forming cocultures. Interestingly, when kif13B decreases, Dlg1 and Sec8 expression levels are also reduced, thus providing additional evidence of the functional relevance of these interactions in myelination. Since Dlg1 interacts with Sec8, which has been shown to mediate membrane formation in oligodendrocytes (Anitei et al., 2006), we hypothesized that the loss of Sec8 function on membrane formation might also contribute to this effect. Indeed, reduction in myelination was also observed by shRNA-mediated depletion of Sec8 in myelin-forming cocultures. We therefore propose a model whereby the kinesin motor protein kif13B transports the multidomain scaffold Dlg1 to sites of membrane remodeling. Interaction of Dlg1 with the Sec 8 exocyst component and with Mtmr2 coordinates a homeostatic control of myelination. Membrane homeostasis is achieved when Sec8-mediated membrane addition and Mtmr2-mediated negative control of membrane formation are properly balanced (Fig. 9). Redundant folds of excessive myelin arise as a consequence of the loss of the Mtmr2-mediated negative control on the amount of new membrane formed via the exocytic machinery. Consistently, downregulation of Sec8 expression in Mtmr2-null cocultures partially rescues myelin outfoldings. Whether kif13B/Dlg1 complex transports Mtmr2 and Sec8 or clusters and concentrates these molecules at sites of membrane remodeling remains to be demonstrated as well as the nature of the cargo of the kif13B/Dlg1-mediated transport. Myelin protein components or junctional proteins of noncompact myelin such as nodal-paranodal regions might represent the cargos. However, several constituents of thigh, adherens, and gap junctions have been found normally localized and expressed in Mtmr2-null nerves (A. Bolis, unpublished data).

Several findings might support our model. In Drosophila, during larvae development, DLG promotes membrane addition by interaction with GTX, a t-SNARE protein involved in membrane fusion, the downstream event that follows Sec8 and exocyst membrane vesicle tethering (Gorczyca et al., 2007). In this system, proper levels of DLG and GTX are required. A decrease of both DLG and GTX results in a decrease of the number of buttons at neuromuscular junctions, whereas their complete loss is embryonically lethal. However, overexpression of DLG sequesters GTX in intracellular structures, whereas overexpression of GTX results in ectopic highly folded membranous structures and infoldings and gives rise to dominant-negative phenotypes. Re-
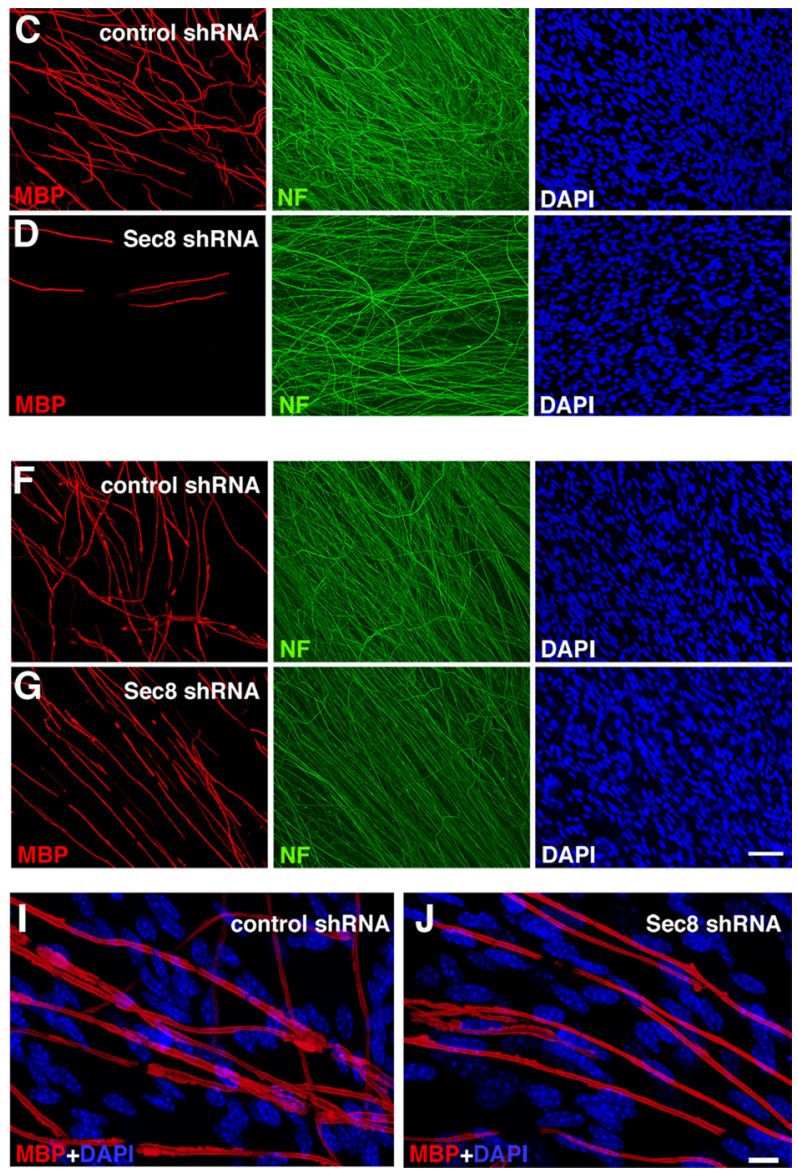

Figure 8. Sec8 is involved in myelin formation in Schwann cells. $A-D, A$ significant decrease in myelination was observed in that did not affect myelination was chosen to transduce Mtmr2-null dissociated cocultures. $\boldsymbol{H}-\boldsymbol{J}$, Myelin outfoldings were significantly rescued also when myelination is not impaired (Sec8 shRNA, $51.79 \pm 1.67, n=6$; control shRNA, $64.78 \pm 3.22, n=6$; $p=0.00716$ ). Error bars indicate SEM. * $p<0.05$. Scale bars: (in $\mathbf{G}) \mathbf{C}, \mathbf{D}, \boldsymbol{F}, \mathbf{G}, 50 \mu \mathrm{m}$; (in J) $\boldsymbol{I}, \boldsymbol{J}, 10 \mu \mathrm{m}$.

cently, it has been also demonstrated that the loss of Dlg1 impairs dendrite formation in vivo, thus providing additional evidence that Dlg1 is involved in membrane formation and growth during development (Zhou et al., 2008).

But how does the phosphatase activity of Mtmr2 contribute to the control of membrane formation during myelination? It is likely that the Mtmr2 phosphatase activity on phospholipids regulates endocytic trafficking and not Sec8-mediated exocytosis. Sec8-mediated vesicle tethering at the plasma membrane is a process promoted by $\operatorname{PtdIns}(4,5) \mathrm{P}_{2}$, the main phospholipid enriched at the plasma membrane (He et al., 2007; Liu et al., 2007; Zhang et al., 2008). Most cell surface $\operatorname{PtdIns}(4,5) \mathrm{P}_{2}$ is generated from PtdIns(4)P (Di Paolo and De Camilli, 2006). Mtmr2 has been shown to dephosphorylate PtdIns(3)P and PtdIns(3,5) $\mathrm{P}_{2}$ (Robinson and Dixon, 2006; Bolis et al., 2007; Nicot and Laporte, 2008), and it is unlikely that the 3-phosphatase activity of Mtmr2 might generate $\operatorname{PtdIns}(4,5) \mathrm{P}_{2}$.

However, PtdIns(3)P and PtdIns $(3,5) \mathrm{P}_{2}$ are involved in the regulation of sorting and membrane dynamics at the level of both early endosomes and late endosomal compartments (Petiot et al., 2003; Michell et al., 2006). It has been recently demonstrated that small interfering RNA-mediated depletion of MTMR2 in A431 cells provokes accumulation of PtdIns(3)P levels and blocks EGFR (epidermal growth factor receptor) egress from late endo- 


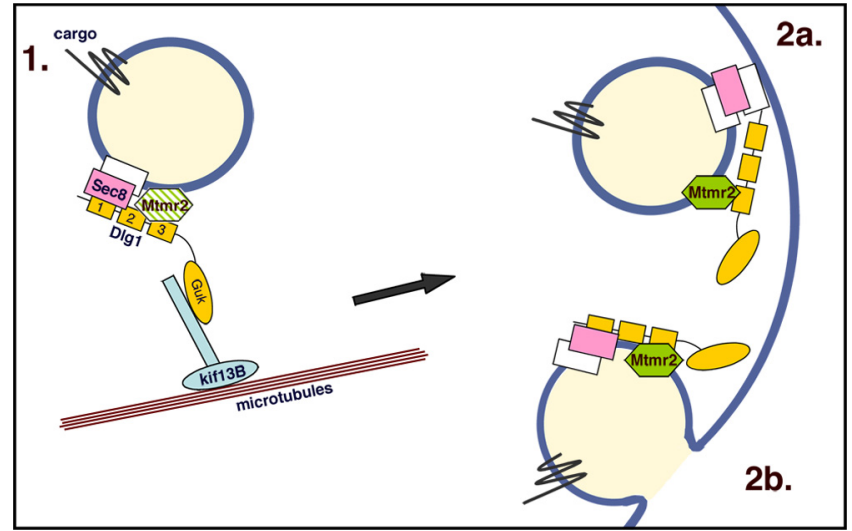

Figure 9. Proposed model of kif13B, Dlg1, Mtmr2, and Sec8 interactions at sites of Schwann cell membrane homeostasis. We propose a model by which Dlg1, transported by kif13B, organizes a molecular platform to coordinate the Sec8-mediated function on membrane formation with that of Mtmr2 on membrane remodeling to achieve homeostasis. Mtmr2 might be either transported via kif13B/Dlg1 (1) or clustered at the sites of membrane homeostasis (2).

somes, where MTMR2 colocalizes with Rab7 (Cao et al., 2008). Thus, Mtmr2 might favor vesicle endocytosis at sites at which Sec8 targets new membranes.

Endocytic recycling allows local remodeling in a variety of cellular processes such as cell morphogenesis during development, biogenesis of epithelial membrane domains, axonal targeting of cell adhesion molecules in neurons, and remodeling of dendritic spines (Lecuit and Pilot, 2003). Recently, the importance of endocytic recycling and of the balance between endocytosis and exocytosis from endosomal pools has been emphasized in myelin-forming oligodendrocytes (Trajkovic et al., 2006; Winterstein et al., 2008).

However, it remains to be assessed whether also in myelinforming Schwann cells MTMR2 dephosphorylates phospholipids involved in endocytosis and endosomal trafficking. It is intriguing that, in skeletal muscle, the overexpression of Mtm1, highly homologous to Mtmr2, provokes packed intracellular membrane structures, which might be caused by excessive caveolae or endocytic vesicle internalization (Buj-Bello et al., 2008).

In conclusion, we here provide evidence for the first molecular mechanism that titrates membrane formation during Schwann cell myelination. We propose a model whereby the kinesin motor protein kif13B transports the multidomain scaffold Dlg1 to sites of membrane remodeling (Fig. 9). Interaction of Dlg1 with the Sec8 exocyst component and with Mtmr2 coordinates a homeostatic control of myelination. Membrane homeostasis is achieved when Sec8-mediated membrane addition and Mtmr2-mediated negative control of membrane formation are properly balanced. Redundant folds of excessive myelin in Mtmr2-null nerves arise as a consequence of the loss of negative control on the amount of membrane formed via Sec8-mediated membrane addition. Consistently, myelin outfoldings are also rescued by reducing Sec 8 expression in Schwann cells.

\section{References}

Anitei M, Ifrim M, Ewart MA, Cowan AE, Carson JH, Bansal R, Pfeiffer SE (2006) A role for Sec8 in oligodendrocyte morphological differentiation. J Cell Sci 119:807-818.

Asaba N, Hanada T, Takeuchi A, Chishti AH (2003) Direct interaction with a kinesin-related motor mediates transport of mammalian discs large tumor suppressor homologue in epithelial cells. J Biol Chem 278:8395-8400.

Biffi A, Capotondo A, Fasano S, del Carro U, Marchesini S, Azuma H, Malaguti MC, Amadio S, Brambilla R, Grompe M, Bordignon C, Quat- trini A, Naldini L (2006) Gene therapy of metachromatic leukodystrophy reverses neurological damage and deficits in mice. J Clin Invest 116:3070-3082.

Bolino A, Muglia M, Conforti FL, LeGuern E, Salih MA, Georgiou DM, Christodoulou K, Hausmanowa-Petrusewicz I, Mandich P, Schenone A, Gambardella A, Bono F, Quattrone A, Devoto M, Monaco AP (2000) Charcot-Marie-Tooth type $4 \mathrm{~B}$ is caused by mutations in the gene encoding myotubularin-related protein-2. Nat Genet 25:17-19.

Bolino A, Bolis A, Previtali SC, Dina G, Bussini S, Dati G, Amadio S, Del Carro U, Mruk DD, Feltri ML, Cheng CY, Quattrini A, Wrabetz L (2004) Disruption of Mtmr2 produces CMT4B1-like neuropathy with myelin outfolding and impaired spermatogenesis. J Cell Biol 167:711-721.

Bolis A, Coviello S, Bussini S, Dina G, Pardini C, Previtali SC, Malaguti M, Morana P, Del Carro U, Feltri ML, Quattrini A, Wrabetz L, Bolino A (2005) Loss of Mtmr2 phosphatase in Schwann cells but not in motor neurons causes Charcot-Marie-Tooth type 4B1 neuropathy with myelin outfoldings. J Neurosci 25:8567-8577.

Bolis A, Zordan P, Coviello S, Bolino A (2007) Myotubularin-related (MTMR) phospholipid phosphatase proteins in the peripheral nervous system. Mol Neurobiol 35:308-316.

Buj-Bello A, Fougerousse F, Schwab Y, Messaddeq N, Spehner D, Pierson CR, Durand M, Kretz C, Danos O, Douar AM, Beggs AH, Schultz P, Montus M, Denèfle P, Mandel JL (2008) AAV-mediated intramuscular delivery of myotubularin corrects the myotubular myopathy phenotype in targeted murine muscle and suggests a function in plasma membrane homeostasis. Hum Mol Genet 17:2132-2143.

Cao C, Backer JM, Laporte J, Bedrick EJ, Wandinger-Ness A (2008) Sequential actions of myotubularin lipid phosphatases regulate endosomal PI(3)P and growth factor receptor trafficking. Mol Biol Cell 19:3334-3346.

Di Paolo G, De Camilli P (2006) Phosphoinositides in cell regulation and membrane dynamics. Nature 443:651-657.

Elias GM, Nicoll RA (2007) Synaptic trafficking of glutamate receptors by MAGUK scaffolding proteins. Trends Cell Biol 17:343-352.

Follenzi A, Naldini L (2002) HIV-based vectors. Preparation and use. Methods Mol Med 69:259-274.

Gerges NZ, Backos DS, Rupasinghe CN, Spaller MR, Esteban JA (2006) Dual role of the exocyst in AMPA receptor targeting and insertion into the postsynaptic membrane. EMBO J 25:1623-1634.

Gorczyca D, Ashley J, Speese S, Gherbesi N, Thomas U, Gundelfinger E, Gramates LS, Budnik V (2007) Postsynaptic membrane addition depends on the Discs-Large-interacting t-SNARE Gtaxin. J Neurosci 27:1033-1044.

Hanada T, Lin L, Tibaldi EV, Reinherz EL, Chishti AH (2000) GAKIN, a novel kinesin-like protein associates with the human homologue of the Drosophila discs large tumor suppressor in T lymphocytes. J Biol Chem 275:28774-28784.

He B, Xi F, Zhang J, TerBush D, Zhang X, Guo W (2007) Exo70p mediates the secretion of specific exocytic vesicles at early stages of the cell cycle for polarized cell growth. J Cell Biol 176:771-777.

Horiguchi K, Hanada T, Fukui Y, Chishti AH (2006) Transport of PIP3 by GAKIN, a kinesin-3 family protein, regulates neuronal cell polarity. J Cell Biol 174:425-436.

Hsu SC, TerBush D, Abraham M, Guo W (2004) The exocyst complex in polarized exocytosis. Int Rev Cytol 233:243-265.

Inoue M, Chang L, Hwang J, Chiang SH, Saltiel AR (2003) The exocyst complex is required for targeting of Glut4 to the plasma membrane by insulin. Nature 422:629-633.

Inoue M, Chiang SH, Chang L, Chen XW, Saltiel AR (2006) Compartmentalization of the exocyst complex in lipid rafts controls Glut4 vesicle tethering. Mol Biol Cell 17:2303-2311.

Kim E, Sheng M (2004) PDZ domain proteins of synapses. Nat Rev Neurosci 5:771-781.

Lecuit T, Pilot F (2003) Developmental control of cell morphogenesis: a focus on membrane growth. Nat Cell Biol 5:103-108.

Lee OK, Frese KK, James JS, Chadda D, Chen ZH, Javier RT, Cho KO (2003) Discs-Large and Strabismus are functionally linked to plasma membrane formation. Nat Cell Biol 5:987-993.

Liu J, Zuo X, Yue P, Guo W (2007) Phosphatidylinositol 4,5-bisphosphate mediates the targeting of the exocyst to the plasma membrane for exocytosis in mammalian cells. Mol Biol Cell 18:4483-4492.

Michell RH, Heath VL, Lemmon MA, Dove SK (2006) Phosphatidylinositol 
3,5-bisphosphate: metabolism and cellular functions. Trends Biochem Sci 31:52-63.

Nakagawa T, Futai K, Lashuel HA, Lo I, Okamoto K, Walz T, Hayashi Y, Sheng M (2004) Quaternary structure, protein dynamics, and synaptic function of SAP97 controlled by L27 domain interactions. Neuron 44:453-467.

Nicot AS, Laporte J (2008) Endosomal phosphoinositides and human diseases. Traffic 9:1240-1249.

Okada Y, Yamazaki H, Sekine-Aizawa Y, Hirokawa N (1995) The neuronspecific kinesin superfamily protein KIF1A is a unique monomeric motor for anterograde axonal transport of synaptic vesicle precursors. Cell 81:769-780.

Petiot A, Faure J, Stenmark H, Gruenberg J (2003) PI3P signaling regulates receptor sorting but not transport in the endosomal pathway. J Cell Biol 162:971-979.

Previtali SC, Nodari A, Taveggia C, Pardini C, Dina G, Villa A, Wrabetz L, Quattrini A, Feltri ML (2003a) Expression of laminin receptors in Schwann cell differentiation: evidence for distinct roles. J Neurosci 23:5520-5530.

Previtali SC, Zerega B, Sherman DL, Brophy PJ, Dina G, King RH, Salih MM, Feltri L, Quattrini A, Ravazzolo R, Wrabetz L, Monaco AP, Bolino A (2003b) Myotubularin-related 2 protein phosphatase and neurofilament light chain protein, both mutated in CMT neuropathies, interact in peripheral nerve. Hum Mol Genet 12:1713-1723.

Previtali SC, Quattrini A, Bolino A (2007) Charcot-Marie-Tooth type 4B demyelinating neuropathy: deciphering the role of MTMR phosphatases. Expert Rev Mol Med 9:1-16.

Robinson FL, Dixon JE (2006) Myotubularin phosphatases: policing 3-phosphoinositides. Trends Cell Biol 16:403-412.

Sans N, Prybylowski K, Petralia RS, Chang K, Wang YX, Racca C, Vicini S, Wenthold RJ (2003) NMDA receptor trafficking through an interaction between PDZ proteins and the exocyst complex. Nat Cell Biol 5:520-530.

Sherman DL, Brophy PJ (2005) Mechanisms of axon ensheathment and myelin growth. Nat Rev Neurosci 6:683-690.

Shevchenko A, Wilm M, Vorm O, Mann M (1996) Mass spectrometric se- quencing of proteins silver-stained polyacrylamide gels. Anal Chem 68:850-858.

Simons M, Trotter J (2007) Wrapping it up: the cell biology of myelination. Curr Opin Neurobiol 17:533-540.

Taveggia C, Zanazzi G, Petrylak A, Yano H, Rosenbluth J, Einheber S, Xu X, Esper RM, Loeb JA, Shrager P, Chao MV, Falls DL, Role L, Salzer JL (2005) Neuregulin-1 type III determines the ensheathment fate of axons. Neuron 47:681-694.

Trajkovic K, Dhaunchak AS, Goncalves JT, Wenzel D, Schneider A, Bunt G, Nave KA, Simons M (2006) Neuron to glia signaling triggers myelin membrane exocytosis from endosomal storage sites. J Cell Biol 172:937-948.

Trapp BD, Kidd GJ (2004) Structure of the myelinated axon. In: Myelin biology and disorders (Lazzarini RA, ed), pp 3-22. London: Elsevier Academic.

Trapp BD, Pfeiffer SE, Anitei M, Kidd GJ (2004) Cell biology of myelin assembly. In: Myelin biology and disorders (Lazzarini RA, ed), pp 29-48. London: Elsevier Academic.

Winterstein C, Trotter J, Kramer-Albers EM (2008) Distinct endocytic recycling of myelin proteins promotes oligodendroglial membrane remodeling. J Cell Sci 121:834-842.

Yamada KH, Hanada T, Chishti AH (2007) The effector domain of human Dlg tumor suppressor acts as a switch that relieves autoinhibition of kinesin-3 motor GAKIN/KIF13B. Biochemistry 46:10039-10045.

Zhang X, Orlando K, He B, Xi F, Zhang J, Zajac A, Guo W (2008) Membrane association and functional regulation of Sec3 by phospholipids and Cdc42. J Cell Biol 180:145-158.

Zhang Y, Luan Z, Liu A, Hu G (2001) The scaffolding protein CASK mediates the interaction between rabphilin3a and beta-neurexins. FEBS Lett 497:99-102.

Zhou W, Zhang L, Guoxiang X, Mojsilovic-Petrovic J, Takamaya K, Sattler R, Huganir R, Kalb R (2008) GluR1 controls dendrite growth through its binding partner, SAP97. J Neurosci 28:10220-10233. 